\title{
INFRARED TRANSMISSION SPECTROSCOPY OF THE EXOPLANETS HD 209458b AND XO-1b USING THE WIDE FIELD CAMERA-3 ON THE HUBBLE SPACE TELESCOPE
}

\author{
Drake Deming $^{1,2}$, Ashlee Wilkins ${ }^{1}$, Peter McCullough ${ }^{3}$, Adam Burrows $^{4}$, Jonathan J. Fortney ${ }^{5}$, Eric Agol ${ }^{2,6}$, \\ Ian Dobbs-Dixon $^{2,6}$, Nikku Madhusudhan ${ }^{7}$, Nicolas Crouzet ${ }^{3}$, Jean-Michel Desert ${ }^{8,17}$, Ronald L. Gilliland ${ }^{9}$, \\ Korey Haynes $^{10,11}$, Heather A. Knutson ${ }^{8}$, Michael Line $^{8}$, ZaZralt Magic $^{12,13}$, Avi M. Mandell ${ }^{11}$, Sukrit Ranjan ${ }^{14}$, \\ David Charbonneau ${ }^{14}$, Mark Clampin ${ }^{11}$, Sara Seager ${ }^{15}$, and Adam P. Showman ${ }^{16}$ \\ ${ }^{1}$ Department of Astronomy, University of Maryland, College Park, MD 20742, USA; ddeming @ astro.umd.edu \\ ${ }^{2}$ NASA Astrobiology Institute's Virtual Planetary Laboratory \\ ${ }^{3}$ Space Telescope Science Institute, Baltimore, MD 21218, USA \\ ${ }^{4}$ Department of Astrophysical Sciences, Princeton University, Princeton, NJ 08544-1001, USA \\ ${ }^{5}$ Department of Astronomy and Astrophysics, University of California, Santa Cruz, CA 95064, USA \\ ${ }^{6}$ Department of Astronomy, University of Washington, Seattle, WA 98195, USA \\ ${ }^{7}$ Yale Center for Astronomy \& Astrophysics, Yale University, New Haven, CT 06511, USA \\ ${ }^{8}$ Division of Geological and Planetary Sciences, California Institute of Technology, Pasadena, CA 91125, USA \\ ${ }^{9}$ Center for Exoplanets and Habitable Worlds, The Pennsylvania State University, University Park, PA 16802, USA \\ ${ }^{10}$ Department of Physics and Astronomy, George Mason University, Fairfax, VA 22030, USA \\ ${ }_{12}^{11}$ NASA's Goddard Space Flight Center, Greenbelt, MD 20771, USA \\ 12 Max-Planck-Institut für Astrophysik, D-85741 Garching, Germany \\ ${ }^{13}$ Research School of Astronomy \& Astrophysics, Weston, ACT 2611, Australia \\ ${ }^{14}$ Harvard-Smithsonian Center for Astrophysics, Cambridge, MA 02138, USA \\ ${ }^{15}$ Department of Earth, Atmospheric and Planetary Sciences, Massachusetts Institute of Technology, Cambridge, MA 02139, USA \\ ${ }^{16}$ Lunar and Planetary Laboratory, University of Arizona, Tucson, AZ 85721, USA \\ Received 2013 February 5; accepted 2013 July 10; published 2013 August 20
}

\begin{abstract}
Exoplanetary transmission spectroscopy in the near-infrared using the Hubble Space Telescope (HST) NICMOS is currently ambiguous because different observational groups claim different results from the same data, depending on their analysis methodologies. Spatial scanning with HST/WFC3 provides an opportunity to resolve this ambiguity. We here report WFC3 spectroscopy of the giant planets HD 209458b and XO-1b in transit, using spatial scanning mode for maximum photon-collecting efficiency. We introduce an analysis technique that derives the exoplanetary transmission spectrum without the necessity of explicitly decorrelating instrumental effects, and achieves nearly photon-limited precision even at the high flux levels collected in spatial scan mode. Our errors are within 6\% (XO-1) and 26\% (HD 209458b) of the photon-limit at a resolving power of $\lambda / \delta \lambda \sim 70$, and are better than $0.01 \%$ per spectral channel. Both planets exhibit water absorption of approximately $200 \mathrm{ppm}$ at the water peak near $1.38 \mu \mathrm{m}$. Our result for XO- $1 \mathrm{~b}$ contradicts the much larger absorption derived from NICMOS spectroscopy. The weak water absorption we measure for HD 209458b is reminiscent of the weakness of sodium absorption in the first transmission spectroscopy of an exoplanet atmosphere by Charbonneau et al. Model atmospheres having uniformly distributed extra opacity of $0.012 \mathrm{~cm}^{2} \mathrm{~g}^{-1}$ account approximately for both our water measurement and the sodium absorption. Our results for HD 209458b support the picture advocated by Pont et al. in which weak molecular absorptions are superposed on a transmission spectrum that is dominated by continuous opacity due to haze and/or dust. However, the extra opacity needed for HD 209458b is grayer than for HD 189733b, with a weaker Rayleigh component.
\end{abstract}

Key words: planetary systems - planets and satellites: atmospheres - techniques: photometric - techniques: spectroscopic

Online-only material: color figures

\section{INTRODUCTION}

From the first discovery of transiting extrasolar planets (Charbonneau et al. 2000; Henry et al. 2000), transmission spectroscopy was anticipated as a potential technique to probe their atmospheres (Seager \& Sasselov 2000). Indeed, transmission spectroscopy was used to make the first detection of an exoplanetary atmosphere (Charbonneau et al. 2002), via optical sodium absorption observed using the Hubble Space Telescope (HST), and sodium and potassium absorption measurements are now possible from the ground (e.g., Redfield et al. 2008; Snellen et al. 2009; Sing et al. 2011, 2012). Using HST data, Barman (2007) identified water absorption near $1 \mu \mathrm{m}$ in the giant exoplanet HD 209458b, and Desert et al. (2008) searched for evidence of

\footnotetext{
${ }_{17}^{17}$ Sagan Fellow
}

$\mathrm{TiO} / \mathrm{VO}$ absorption in that planet. Expanding HST transmission spectroscopy to longer infrared (IR) wavelengths, Swain et al. (2008b) obtained results indicating water and methane absorption near $2 \mu \mathrm{m}$ in the giant exoplanet HD 189733b. Similarly, Tinetti et al. (2010) derived water and carbon dioxide absorption near $1.4 \mu \mathrm{m}$ during the transit of XO- $1 \mathrm{~b}$.

Successful transmission spectroscopy of giant exoplanetary atmospheres is a crucial first step toward eventual spectroscopy of a nearby habitable super-Earth using the James Webb Space Telescope (Deming et al. 2009). However, the IR transmission spectroscopy using HST/NICMOS (Swain et al. 2008b; Tinetti et al. 2010) was challenged by Gibson et al. (2011) who emphasize that the reported absorption features are sensitive to corrections for instrumental systematic errors. Moreover, Gibson et al. (2011) argue that corrections for instrumental error cannot be made using simple linear basis models because 
the "instrument model" is not sufficiently well understood. Crouzet et al. (2012) concluded that NICMOS instrumental signatures remain comparable with the expected amplitude of molecular signatures, even after a decorrelation analysis. New methodology (Gibson et al. 2012b; Waldmann 2012; Waldmann et al. 2013) improves the reliability of NICMOS analyses. However, NICMOS is no longer in operation, so continuing the NICMOS observations per se is not possible.

Fortunately, transmission spectroscopy from 1.1 to $1.7 \mu \mathrm{m}-$ largely overlapping the NICMOS G141 grism region at 1.2-1.8 $\mu \mathrm{m}$-is possible using Wide Field Camera 3 (WFC3) on HST (Berta et al. 2012; Gibson et al. 2012a). Moreover, the WFC3 detector is known to exhibit a more uniform intra-pixel sensitivity response than does NICMOS (McCullough 2008), giving reason to expect that WFC3 observations may be less affected by instrumental signatures. Also, WFC3 can now be operated in a spatial scan mode (McCullough \& MacKenty 2012) wherein the target star is trailed during each exposure by telescope motion perpendicular to the direction of dispersion. Exoplanet host stars are often bright, and the spatial scan allows the longest practical exposures for bright stars without saturating the detector, greatly increasing the overall efficiency of the observations.

In this paper we report WFC3 transmission spectroscopy for two exoplanets observed in our Large (115 orbit) HST Cycle-18 program. By coincidence, these planets (XO-1b and HD 209458b) were both scheduled for observation late in our program, permitting us to acquire the spectra in the newly developed spatial scan mode. One of these planets (XO-1b) is the same as observed in transmission using NICMOS spectroscopy (Tinetti et al. 2010; Gibson et al. 2011; Crouzet et al. 2012). In addition to the great photon-collecting efficiency provided by spatial scan mode, we have achieved some new insights in the analysis of WFC3 data, beyond the valuable methodology introduced by Berta et al. (2012).

We here report robust exoplanetary transmission spectra using WFC3 in spatial scan mode. We achieve a level of precision closely approaching the limit imposed by photon statistics, even for these large exposure levels collected in spatial scan mode. Moreover, our analysis requires no explicit decorrelation using an "instrument model", nor does it require that the pattern of systematic errors be consistent between orbits, or that we omit the first orbit per visit from our analysis.

Section 2 describes the circumstances of our observations, and Section 3 discusses the initial calibration of the data, including a brief discussion concerning the nature of instrumental signatures produced by WFC3. In Section 4 we describe new methodology that we have used to extract the transmission spectra of the planets, and Section 5 gives our results and relates them to previous work. Section 6 interprets our results using model atmospheres for the planets, and Section 7 summarizes and comments on future possibilities.

\section{OBSERVATIONS}

Observations of HD 209458b and XO-1b used WFC3 with the G141 grism, providing wavelength coverage from 1.0 to $1.7 \mu \mathrm{m}$ in first order. Each star was observed during a single visit comprising five consecutive orbits. The observational sequence in each visit began with an undispersed image of the star using the F139M filter (central wavelength of $1390 \mathrm{~nm}$, and FWHM = $32 \mathrm{~nm}$ ). The filter choice for this image is not crucial, because its purpose is merely to establish the position of the undispersed stellar image, used in wavelength calibration.
Table 1

Summary of the Spatial Scan Observations

\begin{tabular}{|c|c|c|}
\hline & $\mathrm{XO}-1 \mathrm{~b}$ & HD $209458 b$ \\
\hline Time of first scan BJD (TDB) & 2455834.6666 & 2456196.0895 \\
\hline Planetary orbital phase at first scan & -0.0471 & -0.0566 \\
\hline Time of last scan BJD (TDB) & 2455834.9419 & 2456196.3997 \\
\hline Planetary orbital phase at last scan & 0.0228 & 0.0314 \\
\hline Number of scans & 128 & 125 \\
\hline Number of $H S T$ orbits & 5 & 5 \\
\hline Scan rate $\left(\operatorname{arcsec~} \mathrm{s}^{-1}\right)\left[\right.$ pixels s$\left.{ }^{-1}\right]$ & $(0.05)[0.41]$ & $(0.9)[7.44]$ \\
\hline Detector subarray size & $128 \times 128$ & $256 \times 256$ \\
\hline Detector reads per scan & 8 & 4 \\
\hline Duration of scan (s) & 50.4 & 32.9 \\
\hline Signal level on detector (electrons pixel ${ }^{-1}$ ) & $3.8 \times 10^{4}$ & $4.4 \times 10^{4}$ \\
\hline
\end{tabular}

Note. Planetary orbital phase is defined to be zero at mid-transit.

Following the undispersed exposure, we obtained a sequence of exposures using the grism, with the star scanned perpendicular to the dispersion, under control of the HST fine-guidance system.

The grism exposures used subarray readouts of the detector to maximize efficiency. Information on the number of exposures, the duration of each sequence, subarray size, and range of planetary orbital phases are given in Table 1. Table 1 also includes the spatial scan parameters and average exposure level in the spectral images, because this information may be useful to subsequent observers.

\section{INITIAL DATA PROCESSING}

WFC3 grism spectroscopy samples the detector "up the ramp", i.e., reading each pixel non-destructively multiple times. We process these data by a method described in the Appendix. After the initial processing, the spectral frames have wavelength in one dimension, with the spatial scan in the orthogonal direction.

Examples of the two-dimensional (2D) spectral frames are shown in Figure 1. One characteristic of spatial scan mode is that the rate of telescope motion is not perfectly uniform, but varies slightly with time due to jitter in the control by the fine guidance system. Evidence of this variation is seen on the rightmost image of Figure 1, which shows the difference between two consecutive scans of HD 209458b. The variable scan rate results in variable flux as a function of row number, typically varying by $\pm 1 \%$ as shown in the plot on Figure 1 . Fortunately, because the scan is perpendicular to the direction of dispersion, this phenomenon does not contribute significant noise to our analysis, but it does affect how we process the data to discriminate against hot pixel and energetic particle hits, as the Appendix describes.

\subsection{Wavelength Calibration and Flat-fielding}

Following the initial data processing described above, we apply wavelength and flat-field calibrations. Each visit includes an undispersed image of the star, and the wavelengths in the grism spectrum are reliably fixed relative to the position of the undispersed image. We calculate those wavelengths using coefficients recommended by STScI (Kuntschner et al. 2009), with some modifications. A. Wilkins et al. (2013, in preparation) found that the original recommended coefficients did not produce optimal agreement between the overall profile of the grism response in observed spectra when compared to 


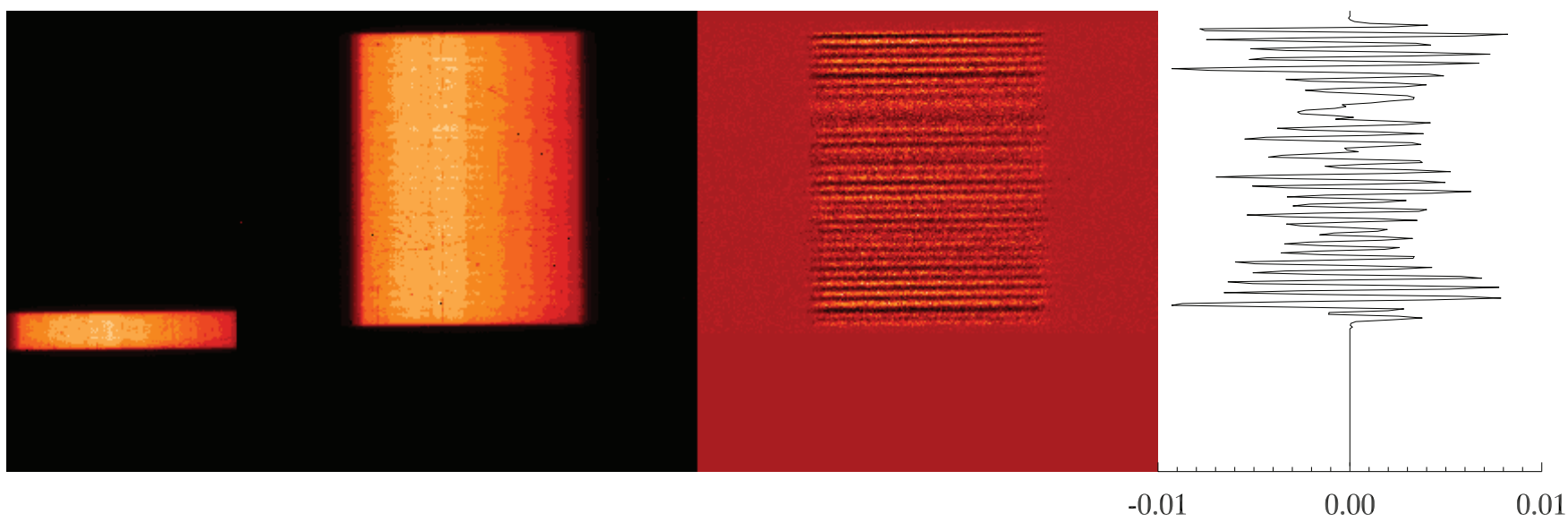

Fractional difference

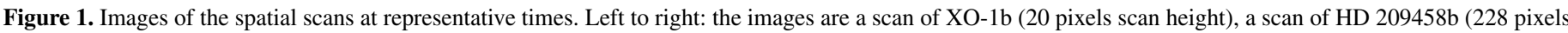

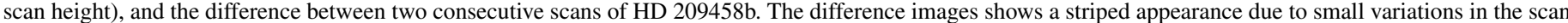

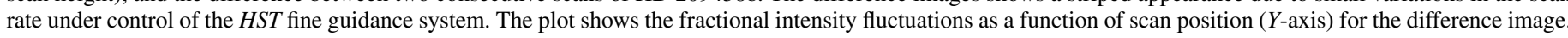
(A color version of this figure is available in the online journal.)

the known profile of that response, and also did not give exact agreement with the known wavelengths of stellar absorption lines. A. Wilkins et al. (2013, in preparation) varied the coefficients by up to $10 \%$ by trial-and-error, to achieve optimal agreement. We have used the A. Wilkins et al. (2013, in preparation) coefficients, and we obtain good consistency of the grism response, and good accounting of stellar absorption lines (e.g., Paschen-beta, see below).

In addition to wavelength calibration, we apply the wavelength-dependent flat-field calibration as recommended by STScI. Note that flat-fielding is not included in STScI pipeline processing, even for the files of non-spatial-scan data, because it is a function of where each target's spectrum happens to fall on the detector, and must be done at the user stage of data analysis. To the extent that the stellar spectra were fixed on the detector during each visit, and jitter and drift in wavelength were negligible, flat-fielding would not be needed in our analysis. In that (ideal) case, we would not apply the flat-fielding step, since it has been our experience that the more the data are processed, the more difficult it becomes to achieve photon-limited results. However, wavelength jitter in spatial scan mode can be larger than in non-scanned observations, so the flat-fielding step of the analysis is prudent. However, we have repeated the entire analysis of this paper without the flat-fielding step, and we find consistent results in the two cases.

\subsection{Instrumental Signatures}

Our analysis is designed to be insensitive to effects caused by the instrument and detector. To understand how we minimize such effects, we must briefly discuss what is known about instrumental signatures in WFC3 G141 grism data. Figure 2 shows a portion of the transit of WASP-18b, which was observed in our program using non-scanned mode, and which clearly illustrates this effect (our spatial scan data show it less clearly). The dominant systematic error is an increase in intensity during each group of exposures that are obtained between buffer dumps. This pattern is shaped like a " $\Gamma$ ", and is slightly reminiscent of a fish hook. It may be physically similar to the ramp effect seen prominently in Spitzer $8 \mu \mathrm{m}$ data, but the WFC3 time scale is shorter and we cannot be certain that it has the same

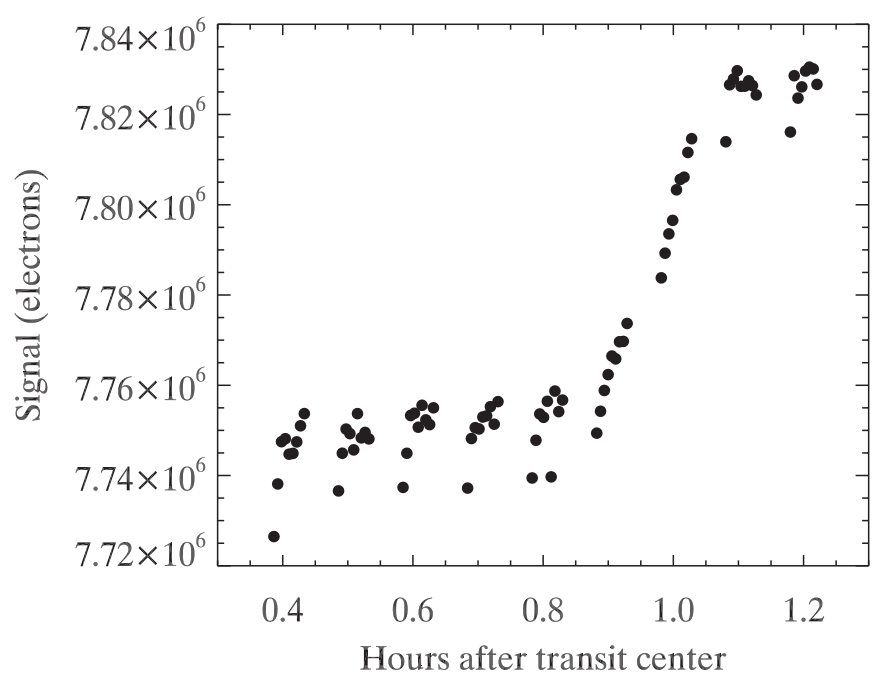

Figure 2. Example of the most prominent instrument-related systematic effect in staring-mode data from our Cycle-18 WFC3 program. This example zoomsin on the egress portion of a transit of WASP-18b, using the integral intensity over each grism spectrum. The space between groups of observed points is due to the time needed to transfer the data buffer. Within each group of points, the intensity increases in a pattern shaped somewhat like " $\Gamma$ ". We call this pattern the "hook".

physical cause as the Spitzer ramp. We therefore use different terminology, and refer to this WFC3 phenomenon as the "hook".

The amplitude of the hook has been studied as a function of exposure level by A. Wilkins et al. (2013, in preparation). They find that the hook is, on average, zero when the exposure level per frame is less than about 30,000 electrons pixel ${ }^{-1}$. Above that exposure level, the amplitude of the hook increases with greater exposure levels, albeit with relatively large scatter in the relation. Our exposure levels are about 40,000 electrons pixel ${ }^{-1}$ (Table 1). Based on the A. Wilkins et al. (2013, in preparation) results, we expect the hook to be weakly detectable in our data.

\section{DERIVATION OF THE EXOPLANETARY SPECTRA}

The principle of our analysis is that we first define and fit the transit as observed in the integral intensity of the grism spectrum over wavelength. Our subsequent analysis 
removes this "white-light" transit, and solves for differential transit depths as a function of wavelength. We construct the exoplanetary transmission spectrum by adding these differential amplitudes to the white-light amplitude to produce the transit depth versus wavelength. There is precedent for this method, from Richardson et al. (2007). In what follows, we describe the steps of our analysis in detail, but we also provide a concise summary in Section 4.7.

Our work has benefited from experience and analyses of nonscanned data in our program (M. Line et al. 2013, in preparation; Mandell et al. 2013; Ranjan et al. 2013; A. Wilkins et al. 2013 , in preparation), as well as the analyses by Berta et al. (2012) and Gibson et al. (2012a). Some of the lessons learned in those analyses are that the hook is usually common-mode to different wavelengths and will cancel in an appropriate ratio. Also, derivation of the exoplanetary spectrum at the edges of the bandpass can be problematic for two reasons. First, the grism spectrum exhibits jitter in wavelength that especially affects the strongly sloped edges of the band and must be corrected as part of the analysis. Second, the intensities decrease at the edges of the grism response, so the amplitude of the hook for pixels sensing those wavelengths will be less, and potentially not common-mode with the remainder of the spectrum.

Following the application of wavelength and flat-field calibration to the 2D spectral frames, we sum each spectral frame spatially (i.e., along columns) to derive one-dimensional (1D) spectra. Mindful of the experience related above, we perform the spatial sum over a range of rows slightly less than the full spatial extent of the scan. In this manner, we avoid including lower intensities that occur at the edges of the scan. We sum in wavelength over a range that exceeds the region of significant grism response, and we restrict the wavelength range (and thus intensity range) in a later step of the analysis (see below). We include the first orbit of each visit in our analysis, but as a precaution we omit the first five frames of the first orbits, where the hook is strongest.

\subsection{The White-light Transit}

Figure 3 shows the white-light photometry for our observed transits of each planet. We have not attempted to correct for the hook, using a divide-out-of-transit (divide-oot) procedure as described by Berta et al. (2012). The hook in these spatial scan data is weak and inconsistent (Figure 3), and good fits are possible without making corrections. To fit the whitelight transit of HD 209458, we generate theoretical transit curves using the formulation of Mandel \& Agol (2002), using nonlinear limb darkening coefficients (Claret 2000), calculated from the emergent intensities of a three-dimensional (3D) hydrodynamic model of the stellar atmosphere (Hayek et al. 2012). We integrated the model intensities over our specific WFC3 bandpass, and fitted the results to the nonlinear law to obtain the coefficients. To quantify the degree to which the results for HD 209458 depend on the treatment of limb darkening, we also do the fits using a linear limb-darkening law (Claret \& Bloemen 2011). We find good agreement in results between the linear and nonlinear limb darkening treatment for HD 209458 (see below). Nonlinear coefficients for XO-1 would require more interpolation in the $3 \mathrm{D}$ model atmosphere grids than we prefer, and our observed noise level is higher for XO-1 than for HD 209458. We therefore use linear limb darkening for XO-1. For both HD 209458 and XO-1, we interpolate the linear coefficients based on values from adjacent bandpasses $(J$ and $H$ ).

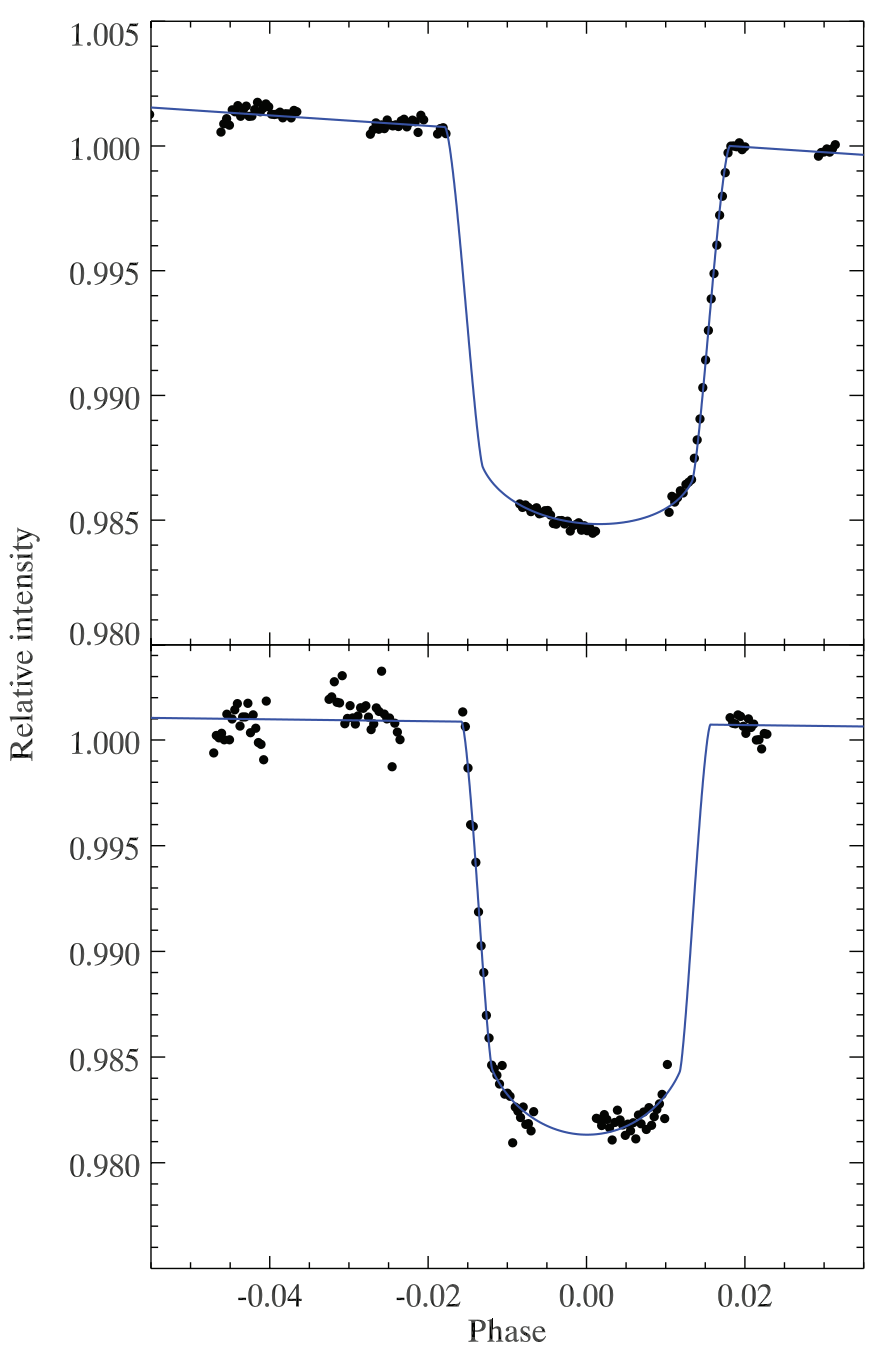

Figure 3. Transits observed in spatial scan mode: HD 209458b (top) and XO-1b (bottom), integrating over the entire grism bandpass ("white light"). No attempt was made to remove systematic effects by divide-oot methodology (Berta et al. 2012); these are purely "as observed". The blue curves are fit to the data by varying $R_{p} / R_{s}$, and a correction to the time of center transit, but fixing other parameters at the values given by Knutson et al. (2007b) and Burke et al. (2010).

(A color version of this figure is available in the online journal.)

For the linear limb darkening coefficients, a range of values are available that vary (typically by \pm 0.03 ) depending on whether they are calculated from PHOENIX versus ATLAS models, and depending on minor parameters such as microturbulence, and on the method used to fit the model atmosphere to the linear law. Our adopted values for linear coefficients correspond to the middle of the range tabulated by Claret \& Bloemen (2011). A similar range of nonlinear coefficients is not available, so we use the variation of the linear coefficient to estimate the impact of limb darkening uncertainties.

For fitting the HD 209458b transit, we adopt the orbital parameters $\left(P, i, a / R_{S}\right)$ from Knutson et al. (2007b), with linear limb darkening coefficient 0.28 . For the nonlinear case we use coefficients calculated for our specific bandpass, as noted above. Our WFC3 observations show a visit-long downward trend. Omitting the first orbit, we are able to fit the remaining data using a linear baseline plus the transit. (Note, however, that we do not omit the first orbit from the spectrum derivation discussed below.) For XO-1b we use the orbital parameters from Burke et al. (2010), with linear limb darkening coefficient of 0.31 . We include the first orbit for XO-1. For each planet, with the 
Table 2

Results for Radius Ratios $\left(R_{p} / R_{s}\right)$ and Mid-transit Times

\begin{tabular}{lcc}
\hline \hline & XO- $1 \mathrm{~b}$ & HD 209458b \\
\hline Mid-transit time BJD (TDB) & $2455834.85186 \pm 0.00017$ & $2456196.28934 \pm 0.00018$ \\
$R_{p} / R_{s}$ & $0.1328 \pm 0.0006$ & $0.1209 \pm 0.0004$ \\
\hline
\end{tabular}

limb darkening and orbital parameters fixed, we vary $R_{p} / R_{s}$ and the time of transit center (to account for imprecision in the ephemeris). The resulting best-fit transit curves are overplotted (blue lines) on Figure 3.

To estimate the errors on our derived values, we use a residual permutation ("prayer-bead") method (Gillon et al. 2007). Also, we manually vary the linear limb darkening coefficient over the range tabulated by Claret \& Bloemen (2011), and note the impact on $R_{p} / R_{s}$. Our adopted errors are the quadrature sum of the variation as a function of linear limb-darkening coefficient, and the prayer-bead errors. For HD $209458 \mathrm{~b}$ we derive $R_{p} / R_{s}=0.1209 \pm 0.0005$ using nonlinear limb darkening, and $R_{p} / R_{s}=0.1214 \pm 0.0005$ using linear limb darkening. Within the errors, our results agree with other IR transit results. Crossfield et al. (2012) derived $R_{p} / R_{s}=0.1218 \pm 0.0014$ at $24 \mu \mathrm{m}$ from several Spitzer transits. For XO-1b, we derive $R_{p} / R_{s}=0.1328 \pm 0.0006$, which agrees (within the errors) with a transit observed using NICMOS by Burke et al. (2010) $\left(R_{p} / R_{s}=0.1320 \pm 0.0005\right)$. Our retrieved transit times are close (tens of seconds) to the predictions using the orbital parameters cited above. Our results for radius ratio and transit time are summarized in Table 2.

\subsection{Wavelength Shifts}

Upon summing the 2D frames in the scan direction to produce 1D spectra, we find that these spectra are not coincident in wavelength as judged by the displacement of the grism response curve. Variations up to \pm 1 column (i.e., in the wavelength direction) occur over the span of each visit, as we measure using least-squares fitting of a template spectrum (see below). For HD 209458, these variations are almost two orders of magnitude larger than similar variations $( \pm 0.02$-columns) seen in non-scanned data. That is reasonable, since it should be much easier for the fine guidance system to hold a fixed position in wavelength when it does not have to scan spatially.

A high quality analysis of spatially scanned data must account for the wavelength shifts. We proceed as follows. First, we construct a template spectrum by averaging spectra that occur before first contact or after fourth contact, by up to one hour. This averaging does not account for the wavelength jitter. Hence, the template spectrum is slightly broadened by the jitter. Restricting the temporal range of the out-of-transit spectra used for the template to within one hour of the contacts, and restricting the wavelength range to avoid the strongly sloping edges of the grism response, we minimize broadening of the template due to the wavelength jitter. Broadening of the template is not detectable in the residuals of our fits.

After forming the template spectrum we shift it in wavelength, and fit it to all of the individual grism spectra in the visit. For each grism spectrum, we step through a large range of wavelength shifts of the template, using 0.001 pixel increments, to assure that the best-fit shift is identified. At each shift value, the best trial fit is achieved by a linear stretch of the template spectrum in intensity (via linear least-squares). No stretching of the template is applied in the wavelength coordinate—only a shift. The fitting

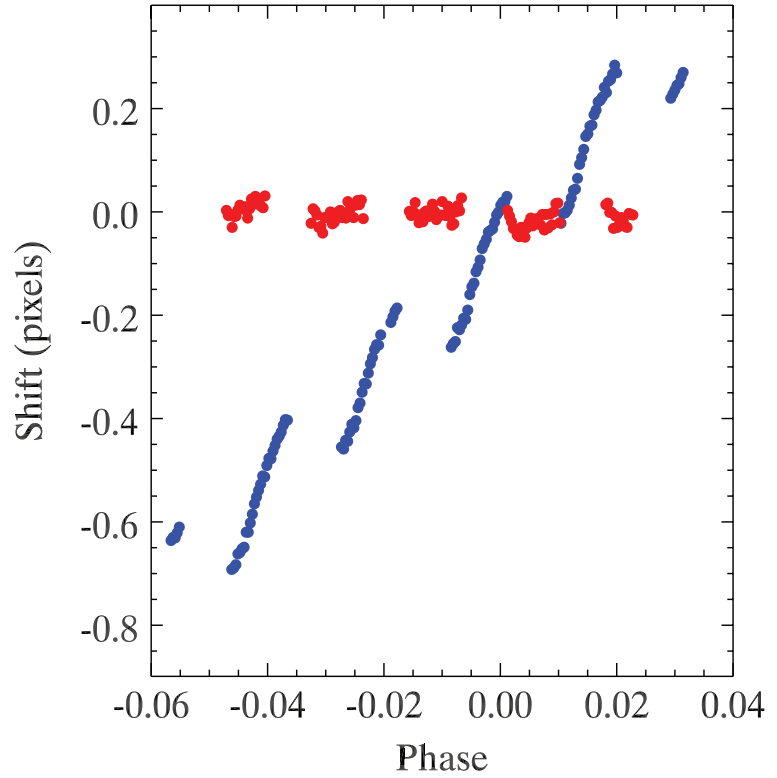

Figure 4. Wavelength shifts derived from our shift-and-fit procedure, vs. orbital phase. The grouping of the points shows the different orbits in each visit. Blue points are HD 209458 and red points are XO-1.

(A color version of this figure is available in the online journal.)

process simultaneously removes residual background intensity in the spectra (see the Appendix). The factor required for the intensity stretch is very closely correlated to the total intensity of the system due to the transit; the stretch factor versus orbital phase closely resembles Figure 3.

After trying the full range of possible wavelength shifts, we pick the best wavelength shift and linear stretch factor based on the minimum $\chi^{2}$. Shifting the template spectrum requires re-sampling it by interpolation. We use the IDL routine INTERPOLATE, with the cubic keyword set to -0.5 , this being the best approximation to ideal interpolation using a sinc function. Because we shift the template to match individual spectra, re-sampling of each individual spectrum is avoided, further minimizing the potential for adding noise via the resampling process. The wavelength shifts (in pixels) that we derive from our fits are shown in Figure 4; the largest shifts are seen for HD 209458b, versus much smaller shifts for XO-1b.

Upon fitting the template spectrum to a given individual spectrum, we subtract them and form residuals $R_{t \lambda}$, where $t$ indexes time (i.e., what individual spectrum), and $\lambda$ indexes wavelength. This subtraction removes the small amount of sky background that survives the process described in the Appendix. This fitting and subtraction is done separately at each time step $t$, and it removes the wavelength variations of the grism response, as well as canceling common-mode systematic errors (see below). Because the flux from the star varies with time due to the transit itself, we include a factor to normalize the $R_{t \lambda}$ in units of the out-of-transit stellar flux. An illustration of the match between an individual spectrum and the template is shown in Figure 5, including the residuals in the lower panel. At each $\lambda$, the time series $R_{t \lambda}$ contain the differential transits that 


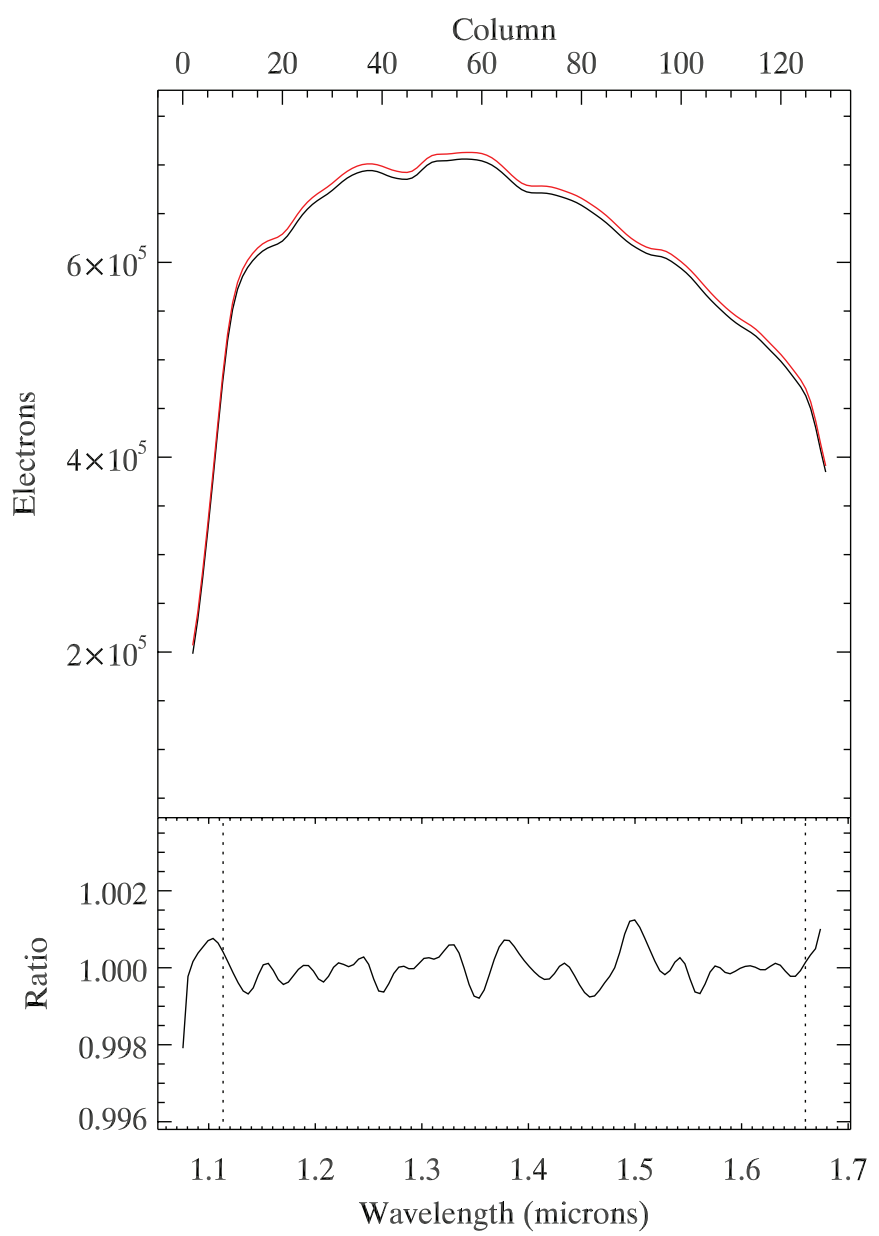

Figure 5. Top panel: example of the grism spectrum of XO-1. The black line is a spectrum at a single time, showing the roll-off in grism response shortward of $1.1 \mu \mathrm{m}$ and longward of $1.65 \mu \mathrm{m}$. The red curve is the out-of-transit template spectrum (see text), shifted upward by $1 \%$ for clarity of illustration. These spectra have been smoothed using a Gaussian kernel having FWHM $=4$ pixels. Lower panel: difference between the single smoothed spectrum and best-fit template, normalized by the intensity in the template spectrum. The vertical dashed lines define the wavelength range used in the analysis of $\mathrm{XO}-1 \mathrm{~b}$. (A similar range was used for HD 209458b.)

(A color version of this figure is available in the online journal.)

we seek. To facilitate cancellation of the hook and potentially other systematic errors, we restrict the intensity range of our analysis - hence the wavelength range - to wavelengths whose intensity lies above the half-power points of the grism response (see dashed lines on Figure 5).

The fitting of the template to form the $R_{t \lambda}$ is key to our analysis, because it helps to cancel common-mode systematic errors. That cancellation is conceptually equivalent to dividing the intensity in the grism spectrum at a given wavelength by the integral of each grism spectrum over wavelength. Thus, our method is similar to the divide-oot procedure used by Berta et al. (2012), but is (arguably) more general. The divide-oot method relies on the pattern of systematic error being consistent in time, whereas we here require that it be common-mode in wavelength.

To see why our procedure described above is equivalent to a wavelength ratio, consider the following. We use a single template spectrum per planet for each visit. We stretch that template spectrum in intensity for the fitting process, but the stretch is a linear factor common to all wavelengths. Therefore, the ratio of the template at a given wavelength to its integral over wavelength is constant. Moreover, the fitting

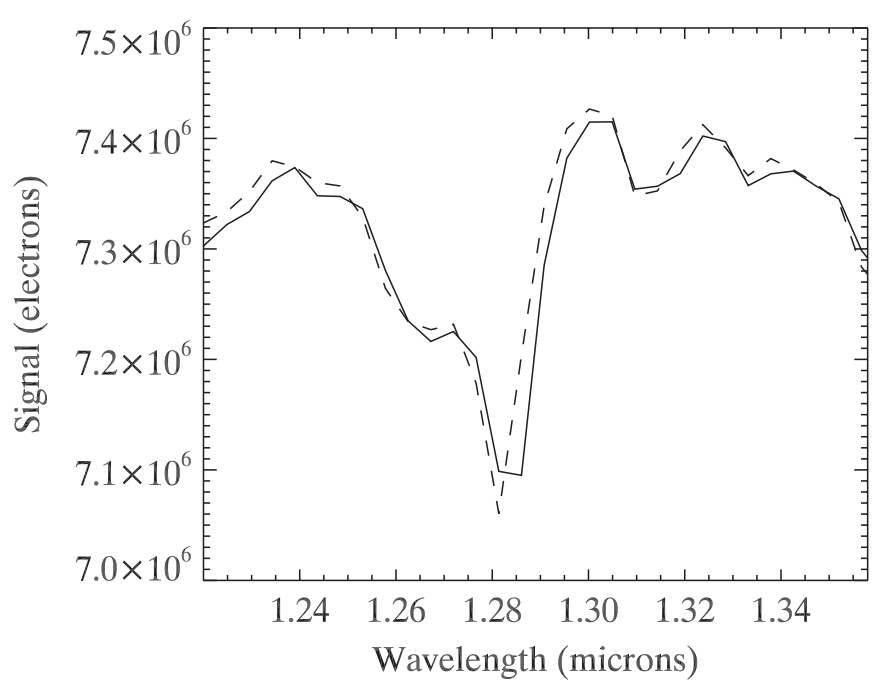

Figure 6. Example of undersampling in the WFC3 spectra. Shown are zoomedin portions of two spectra of HD 209458, separated by $3.1 \mathrm{hr}$ of time (in orbits 1 and 3 of visit 26 in our program). The dip at $1.28 \mu \mathrm{m}$ is the Paschen-beta line in the star. In the earlier spectrum (solid line), the line core appears flattened because the line is positioned mid-way between two columns of the detector. In the later spectrum (dashed line), the line core is sharp because the different overall shift in wavelength places it centered on a column.

process guarantees that the wavelength integral of the stretched template will be closely equal to the wavelength integral of the individual spectrum being fit. Hence, apart from a constant factor, normalizing an individual residual by the value of the template spectrum at that wavelength is conceptually the same as dividing the individual spectrum at that wavelength by its integral over wavelength.

Although we have described our method as being equivalent to a ratio-of-wavelengths, the illumination level of the various pixels is probably a more relevant physical variable than wavelength per se. Since our analysis is restricted to intensities not greatly below the peak of the grism response (Figure 5), the intensities in the data covered by our analysis tend to be restricted to a limited range, and this is probably the dominant factor in cancellation of systematic errors.

Note that our analysis procedure as described above (and further below) does not involve any explicit decorrelation versus instrument or telescope parameters (e.g., tilt of the spectrum, HST orbital phase, detector temperature, etc.). Like the divideoot method (Berta et al. 2012), we rely on cancellation of common-mode systematic errors by operating only on the data themselves, using simple linear procedures.

\subsection{Undersampling}

Initial correction of wavelength shifts using the above procedure showed discordant results at some wavelengths, characterized by strong slopes and even nonlinear temporal trends in the $R_{t \lambda}$ values as a function of $t$. The most discordant results occurred near strong stellar lines such as Paschen-beta $(1.28 \mu \mathrm{m})$. We initially suspected interpolation errors in the shift-and-fit process, but careful inspection of the uninterpolated spectra revealed that the shapes of the stellar lines were changing as a function of the wavelength shift, due to undersampling of the grism resolution by the pixel grid. The FWHM resolution of the G141 grism at $1.28 \mu \mathrm{m}$ equals 2.3 pixels. This is insufficient to eliminate changes in the pixel-sampled line shape as a function of wavelength shift. Figure 6 shows the Paschen-beta line in two spectra separated by about $3 \mathrm{hr}$ in the visit for HD 209458. 
The change in line shape is obvious. This line shape change is not mere noise, since it is consistent over many spectra, and the pixel-sampled line shape changes gradually and smoothly as a function of wavelength shift.

WFC3 sampling of 2.3 pixels per spectral resolution nominally complies with the criterion of the Nyquist-Shannon sampling theorem (Shannon 1949). However, the grism spectral response can violate the premise of the theorem in the sense that its Fourier decomposition may contain components at spatial frequencies higher than the nominal resolution. So the undersampling we infer here is not surprising.

Changes in the shape of pixel-sampled stellar lines during a transit will cause noise that cannot be removed using any simple divide-oot or ratio procedure. Moreover, we cannot change the dimensions of the pixel spacing. Our solution is to force adequate sampling of the spectral resolution, by degrading the resolution post-detection. Prior to the analysis described above (i.e., before forming and using the template spectrum), we convolve each 1D spectrum with a Gaussian kernel having FWHM $=4$ pixels. We varied the width of the kernel to determine the best compromise between suppression of undersampling errors and degradation of the spectral resolution. We apply the convolution to the template spectrum as well as to individual spectra. Figure 5 has been convolved with our adopted kernel. Because of the linearity of our analysis, we arguably could achieve similar results by fitting the template spectrum in the presence of undersampling errors and averaging the resultant exoplanetary spectrum over wavelength in a subsequent step of the analysis. We elect to smooth the grism spectra at an early stage of the analysis because that gives us more insight into the nature of the errors when deriving differential transit amplitudes.

\subsection{Differential Transits}

The wavelength dependence of the transit depths is contained in the $R_{t \lambda}$ residuals. Note that the smoothing procedure described above introduces autocorrelation in the residuals as a function of $\lambda$ (apparent on Figure 5), but not as a function of $t$. At each $\lambda$, we fit a scaled transit curve to the $R_{t \lambda}$, with a linear baseline. The shape of the transit curve is constrained to be the same as the white-light (Figure 3) transit for each planet, except that we include a correction for the wavelength dependence of limb darkening. We allow the amplitude of the fitted curve to vary, since that is essential to deriving the exoplanetary spectrum. The best-fit amplitude and baseline slope are found simultaneously via linear regression. We used two different versions of the linear baseline. First, we used a baseline that is linear as a function of orbital phase (called phase baseline). Second, we used a linear baseline that is linear as a function of the ordinal time step (called ordinal baseline). Our derived exoplanetary transmission spectra are insensitive to the nature of the linear baseline (phase or ordinal), but the ordinal baseline gives about $2 \%$ smaller errors, so we adopt it for our final fits. The slightly lower errors for the ordinal baseline may indicate that the instrument effects not canceled by our shift-and-fit procedure depend on the exposure number to a greater degree than they depend on mere elapsed time.

The baseline slopes retrieved from the regression are modest, and have little impact on our results. We also verified that the slopes are uncorrelated with the derived exoplanetary spectral amplitudes (Pearson correlation coefficients of about 0.15). Note that more sophisticated (e.g., Markov Chain; Ford 2005) techniques would be superfluous in this situation, since we are not concerned with errors introduced by correlations between parameters, etc. Nor do we need to consider uncertainties on priors like the planet's orbital parameters, because the relevant priors are already known to high precision and are not dominant in our analysis. Instead, the dominant problem is simply to find the best-fit differential amplitude and baseline in the presence of noise.

Although we calculate the differential transit amplitudes by fitting a transit curve with a linear baseline, we also checked the results using a much simpler procedure. Dividing the $R_{t \lambda}$ at each $\lambda$ into an in-transit and out-of-transit group, we subtract the average of the out-of-transit residuals from the average of the in-transit residuals at each $\lambda$. This simple in-minus-out procedure yields transmission spectra that are very similar to the more rigorous method of fitting a transit curve (fitting the curve accounts for the ingress and egress portions correctly, and it permits us to correct for the wavelength dependence of limb darkening).

There has been considerable discussion in the literature concerning methodologies to derive exoplanetary transmission spectra (e.g., Gibson et al. 2011; Swain et al. 2008b), including some quite sophisticated techniques (Gibson et al. 2012a, 2012b; Waldmann 2012; Waldmann et al. 2013). While we respect the power of sophisticated analyses, we advocate the virtue of making the signal visible to the eye using the simplest linear processes. To that end, we present Figure 7, which shows the differential transit data for HD 209458b, binned in intervals of four wavelength columns. This is the same binning that we use for our final spectral results. A nominal difference is that our final results come from fitting to single wavelengths, then averaging the differential transit amplitudes (see below), whereas Figure 7 shows fits to the binned data. Since the fitting process is linear (average of the fits equals fit to the average), there is no real difference, and Figure 7 represents our actual results for 10 binned wavelengths spanning the water vapor bandhead in HD 209458b. Notice that as wavelength increases toward the bandhead at $\sim 1.38 \mu \mathrm{m}$, the differential transits change from negative (inverted) or near-zero amplitudes, to deeper-than-average transits that are obvious by eye. Figure 8 shows a similar comparison for $\mathrm{XO}-1 \mathrm{~b}$, but with more wavelength averaging, as appropriate to the lower $\mathrm{S} / \mathrm{N}$ for that planet.

As noted above, we bin the differential transit amplitudes by four columns to be approximately consistent with the smoothing used to suppress the detector undersampling (Section 4.3). The consistency is only approximate because a square-wave binning (four columns exactly), and a Gaussian smoothing produce similar-but not identical-averaging. The wings of the Gaussian kernel used in Section 4.3 extend beyond \pm 2 pixels. Convolving also with the intrinsic 2.3 pixel instrumental FWHM, we calculate that about $15 \%$ of binned channel $N$ spills into binned channel $N+1$, and vice versa. That level of residual autocorrelation is not a significant factor in the interpretation of our current results, but should be borne in mind by future investigators using our methodology. Our derived exoplanetary transmission spectra for HD 209458b and XO-1b are tabulated in Table 3.

\subsection{Verification of Sensitivity}

Anticipating our results (Section 5), we find exoplanetary water absorptions that are of significantly smaller amplitude than previous investigators claim for the same planets. We therefore verified the sensitivity of our analysis by numerically injecting a synthetic signal into our data, and we recovered it with the correct amplitude. Specifically, we added a synthetic transit of 

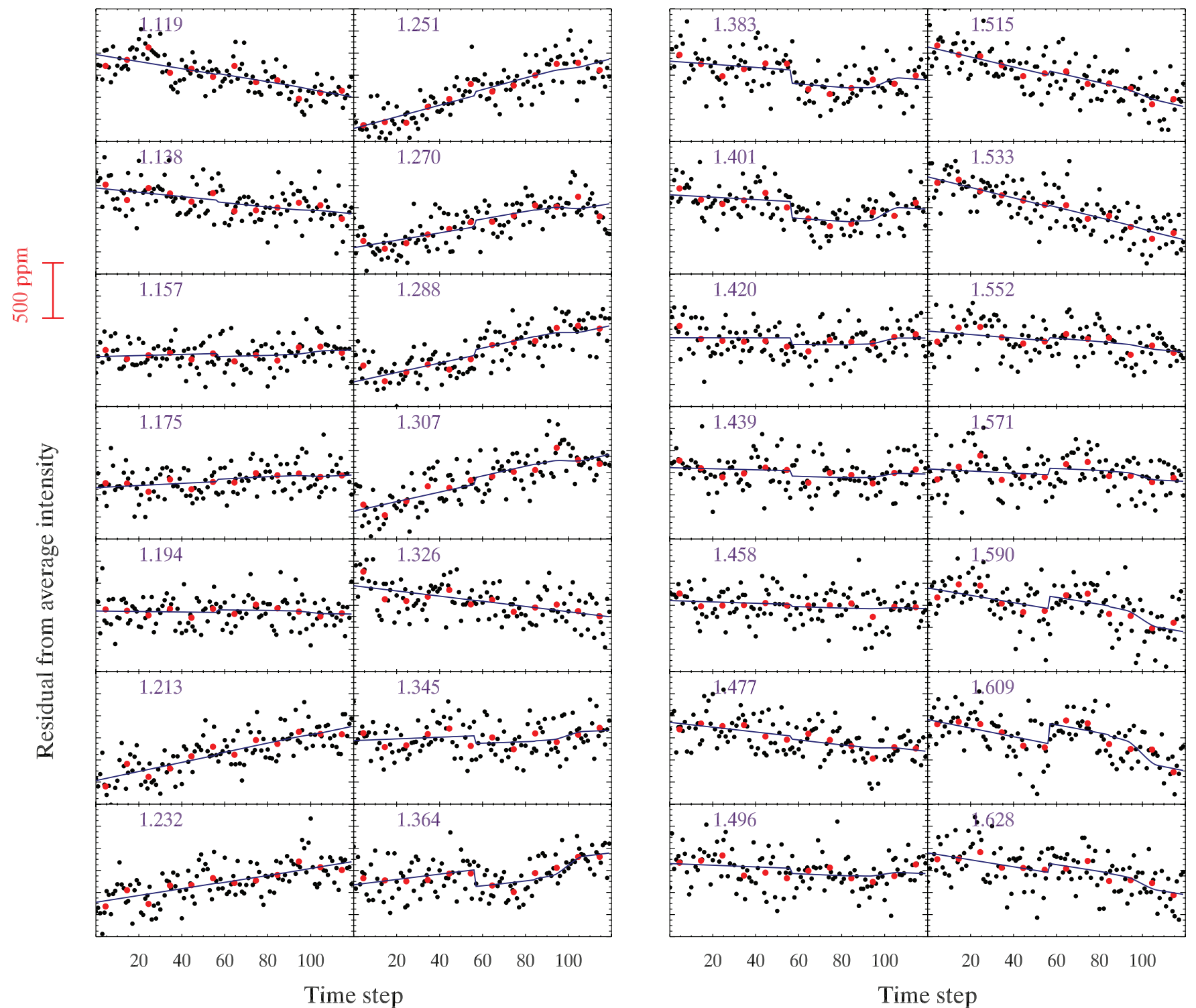

Figure 7. Fits of differential transit curves to spectral intensity residuals for HD 209458b. Plotted are values of residuals $R_{t \lambda}$, binned over four values of $\lambda$. In practice, we fit the differential phase curves to data at individual $\lambda$ values, then we bin the fitted amplitudes to form the transmission spectrum. These panels show the binned data compared to a binning of the fits for the four wavelengths in each bin. Each panel is labeled by binned wavelength in microns. For all panels, the red points are temporal averages of 10 data points and are plotted for illustration purposes only; no temporal averaging is used in the fitting process. The fitted curves (in blue) include a linear baseline as a function of ordinal time step, as well as the differential transit. The differential transit can appear distorted when plotted vs. ordinal time step (see Section 4.4 discussion of ordinal vs. phase baselines), but the transit curve is generated correctly as a function of orbital phase. Note the obvious increase in differential transit depth near the bandhead wavelength at $\sim 1.38 \mu \mathrm{m}$ (compare to upper panel of Figure 10). Also, note that these differential transit depths are illustrated prior to the correction for wavelength-dependent limb darkening. Limb darkening increases transit depths with decreasing wavelength.

(A color version of this figure is available in the online journal.)

amplitude $500 \mathrm{ppm}$, occurring only in 10 columns of the detector spanning wavelengths $1.225-1.272 \mu \mathrm{m}$, to the HD $209458 \mathrm{~b}$ data. We added this synthetic signal immediately after the stage of producing the scanned data frames (Equation (1) in the Appendix). Our analysis retrieved this signal at the full injected amplitude (not illustrated here), with the expected rolloff at the edges of the simulated sharp band due to the smoothing used in our analysis. We conclude that our analysis does not numerically attenuate exoplanetary transmission signals to any significant extent.

\subsection{Errors}

We estimate the errors associated with our differential transit amplitudes using two techniques. First, we calculate the standard deviation of single points in each differential transit curve, after the best-fit differential transit is removed (i.e., in the residuals). We denote this value as $\sigma_{1}$. Then, we bin the residuals of each transit curve over $\mathrm{N}$ points, varying $\mathrm{N}$ up to half the number of observed points in time, and we calculate the standard deviation of each binned set, denoted $\sigma_{N}$. For Poisson errors in the absence of red noise, we expect

$$
\log \left(\sigma_{N}\right)=\log \left(\sigma_{1}\right)-0.5 \log (N)
$$

The slope of the observed $\log \left(\sigma_{1}\right)$ versus $\log \left(\sigma_{N}\right)$ relation is uncertain at a single wavelength due to the paucity of points at high- $N$ (i.e., few large bins). For better statistics, we accumulate the $\sigma$ values over all wavelengths, and show the dependence of $\sigma_{N}$ versus $\log (N)$ on Figure 9 for both planets. The blue lines 


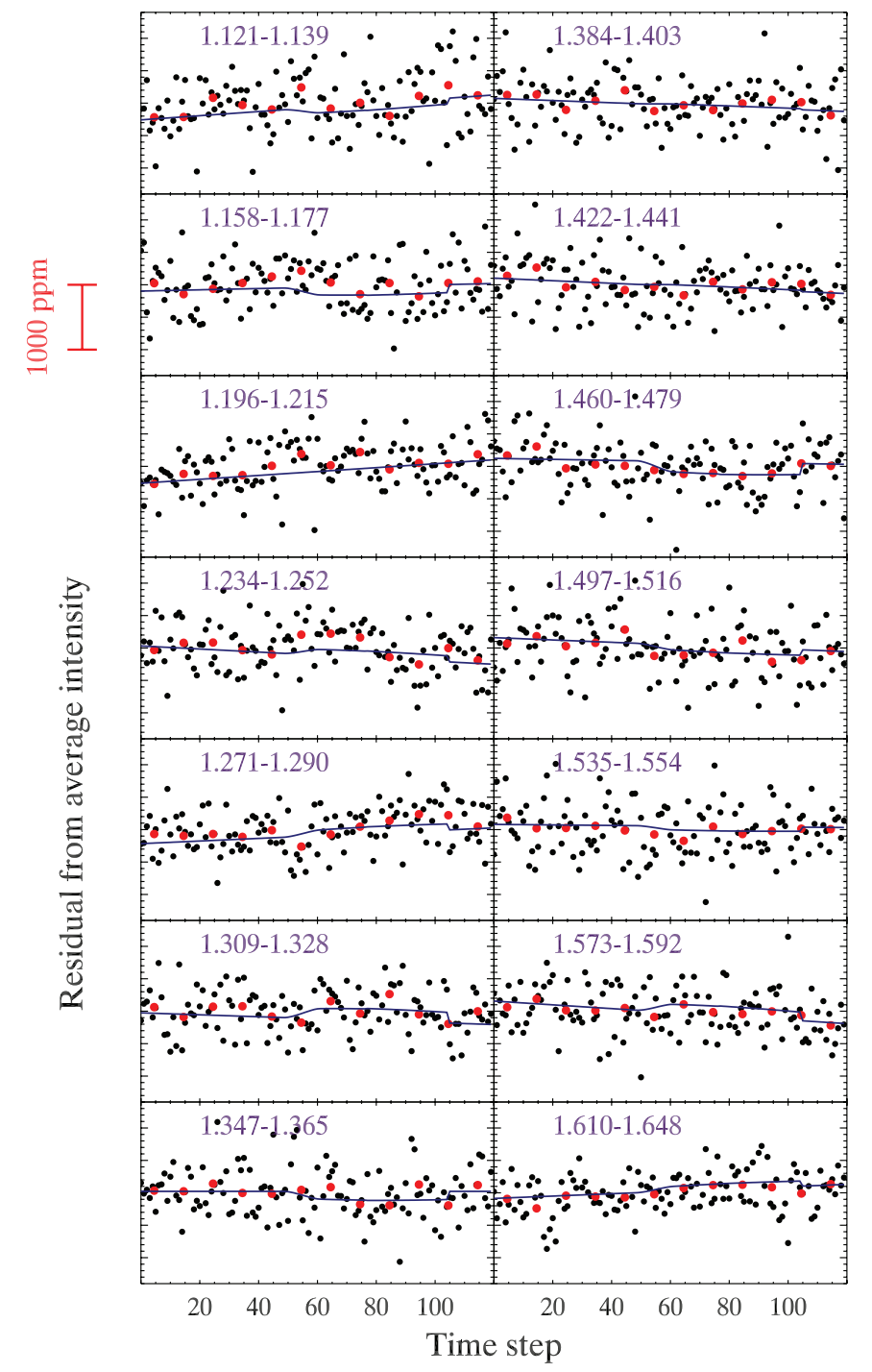

Figure 8. Fits of differential transit curves to spectral intensity residuals for $\mathrm{XO}-1 \mathrm{~b}$ (see caption of Figure 7 for explanation). To reduce the scatter, and improve the clarity for this fainter system, most panels show the average of two wavelength bins (compare to Table 3), except the lower right panel that includes three wavelength bins.

(A color version of this figure is available in the online journal.)

on Figure 9 represent the errors expected in the limit of photon statistical noise, that decreases proportional to $-0.5 \log (N)$. These lines are calculated a priori from the number of electrons in the spectrum, and their close accord with the measured scatter implies that the errors of our analysis are close to photon-limited. Our cumulative $\sigma(N)$ values are in good agreement with the expected photon noise and the slope of -0.5 in Equation (2). One seeming difference on Figure 9 is that both planets exhibit some $\sigma_{N}$ points that scatter well below the photon noise limit at large $N$. This occurs because the differential transit fitting acts as a high-pass filter. Even if the differential transit amplitude is zero, the linear regression will often find a non-zero amplitude due to noise at large bin sizes. The regressions will therefore tend to remove low frequency noise as a byproduct of deriving the differential transit amplitudes.

Based on Figure 9, we calculate the error associated with each differential transit amplitude as being the quadrature sum of the in- and out-of-transit levels in the $R_{t \lambda}$ values, calculating the errors on these levels from Equation (2). We also used the
Table 3

Results for Transmission Spectra

\begin{tabular}{lccccr}
\hline \hline & HD $209458 \mathrm{~b}$ & & & XO-1b & \\
$\lambda$ & $R_{p}^{2} / R_{s}^{2}(\mathrm{ppm})$ & Error $(\mathrm{ppm})$ & $\lambda$ & $R_{p}^{2} / R_{s}^{2}(\mathrm{ppm})$ & Error $(\mathrm{ppm})$ \\
\hline 1.119 & 14512.7 & 50.6 & 1.121 & 17545.5 & 100.4 \\
1.138 & 14546.5 & 35.5 & 1.139 & 17697.6 & 97.6 \\
1.157 & 14566.3 & 35.2 & 1.158 & 17582.1 & 96.7 \\
1.175 & 14523.1 & 34.6 & 1.177 & 17772.4 & 94.8 \\
1.194 & 14528.7 & 34.1 & 1.196 & 17685.8 & 93.4 \\
1.213 & 14549.9 & 33.7 & 1.215 & 17427.6 & 92.3 \\
1.232 & 14571.8 & 33.5 & 1.234 & 17386.4 & 91.6 \\
1.251 & 14538.6 & 33.6 & 1.252 & 17552.8 & 91.6 \\
1.270 & 14522.2 & 33.8 & 1.271 & 17538.6 & 92.0 \\
1.288 & 14538.4 & 33.7 & 1.290 & 17435.2 & 91.5 \\
1.307 & 14535.9 & 33.4 & 1.309 & 17323.6 & 90.8 \\
1.326 & 14604.5 & 33.4 & 1.328 & 17525.0 & 90.7 \\
1.345 & 14685.0 & 33.5 & 1.347 & 17696.1 & 90.7 \\
1.364 & 14779.0 & 33.9 & 1.365 & 17832.1 & 91.4 \\
1.383 & 14752.1 & 34.4 & 1.384 & 17674.6 & 92.6 \\
1.401 & 14788.8 & 34.5 & 1.403 & 17569.4 & 93.0 \\
1.420 & 14705.2 & 34.7 & 1.422 & 17609.2 & 93.2 \\
1.439 & 14701.7 & 35.0 & 1.441 & 17660.1 & 93.8 \\
1.458 & 14677.7 & 35.4 & 1.460 & 17923.9 & 94.7 \\
1.477 & 14695.1 & 35.9 & 1.479 & 17799.7 & 96.1 \\
1.496 & 14722.3 & 36.4 & 1.497 & 17794.9 & 97.3 \\
1.515 & 14641.4 & 36.6 & 1.516 & 17771.4 & 97.9 \\
1.533 & 14676.8 & 37.1 & 1.535 & 17753.9 & 98.7 \\
1.552 & 14666.2 & 37.8 & 1.554 & 17799.1 & 100.4 \\
1.571 & 14642.5 & 38.6 & 1.573 & 17590.7 & 102.4 \\
1.590 & 14594.1 & 39.2 & 1.592 & 17560.9 & 104.0 \\
1.609 & 14530.1 & 39.9 & 1.610 & 17719.4 & 105.5 \\
1.628 & 14642.1 & 40.8 & 1.629 & 17650.2 & 107.7 \\
& & & 1.648 & 17595.9 & 110.6 \\
\hline & & & & &
\end{tabular}

Notes. Wavelength $(\lambda)$ is in $\mu \mathrm{m}$, and transit depth in parts per million (ppm). Note that the tabulated errors apply to the differential transit depths; a larger error applies to radius ratio over the entire range-see Table 2. (Our re-analyzed STIS transit depths are not listed here, but are uniformly $763 \mathrm{ppm}$ larger than given by Knutson et al. (2007b).)

prayer-bead method (Gillon et al. 2007) to calculate the error on each differential transit amplitude. This also indicated close agreement with the photon limit, but the precision of the prayerbead error estimate at a single wavelength is limited by the relatively small number of possible permutations. Therefore we adopted our final errors using the following procedure. For each planet, we calculated the average ratio of the prayer bead to photon errors, and the scatter about this average. We multiply the photon errors by this average ratio to obtain our adopted errors for most wavelength bins. However, a few wavelengths exhibit prayer bead errors more than $3 \sigma$ greater than the average error level. For these points, we use the prayer bead error estimate for that specific wavelength.

Our derived transmission spectra for HD 209458b and XO-1b are shown on Figure 10, and tabulated in Table 3. Our average error bar for the spectrum of HD 209458b is $36 \mathrm{ppm}$, which is 1.26 times the photon noise. The largest error bar for our 28 wavelength channels is $51 \mathrm{ppm}$. For $\mathrm{XO}-1 \mathrm{~b}$, our average errors are $96 \mathrm{ppm}$, which is 1.06 times the photon noise. The largest $\mathrm{XO}-1 \mathrm{~b}$ error bar is $111 \mathrm{ppm}$.

\subsection{Summary of Our Spectral Derivation Methodology}

To summarize our method as described above:

1. From 2D spectral images that are flat-fielded and wavelength-calibrated, we make $1 \mathrm{D}$ grism spectra. We sum 


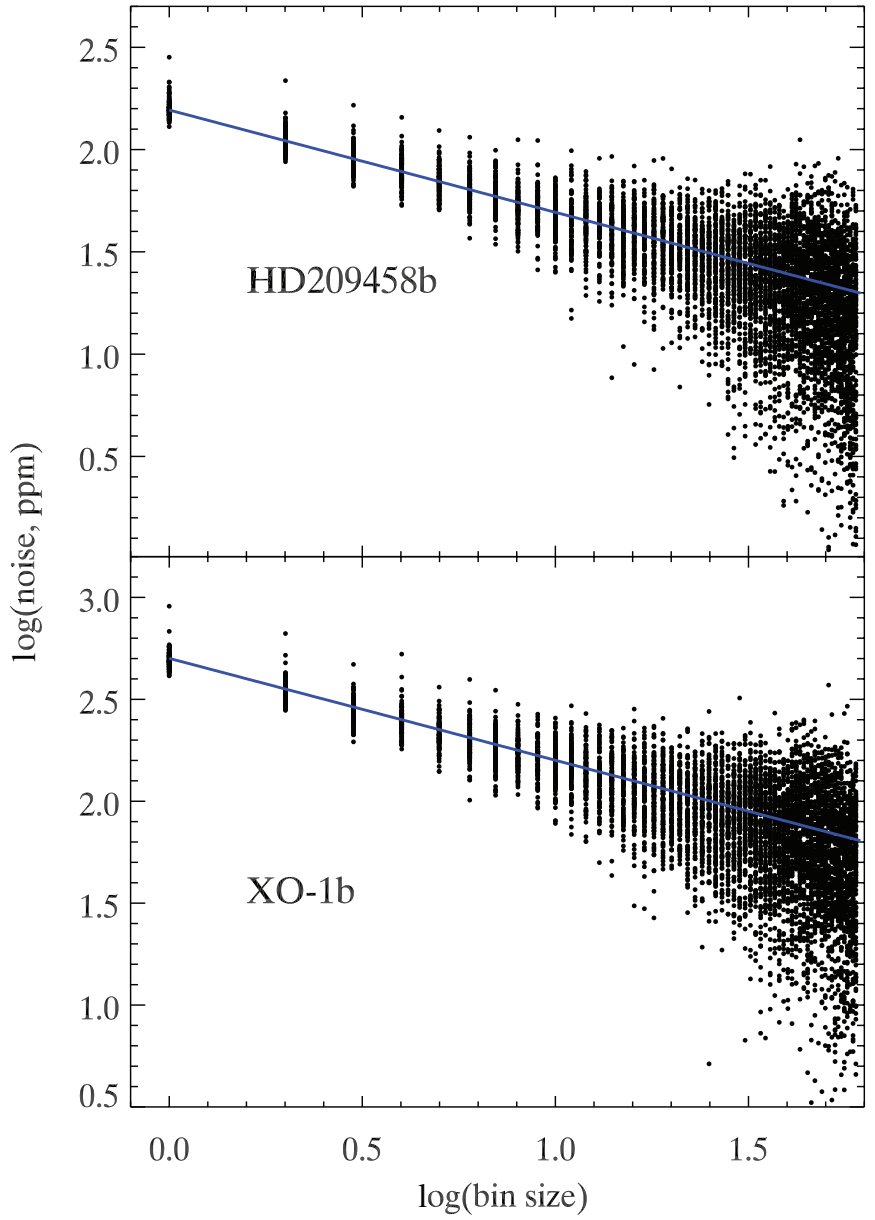

Figure 9. Error analysis for our derived exoplanetary transmission spectra. Each panel plots the standard deviation of the observed noise in our differential transits, after removing the best-fit amplitude. The noise is shown as a function of bin size. The blue lines are the relations expected for photon noise based on the number of detected electrons, and accounting for the effect of smoothing the grism spectra (Section 4.3). The blue lines have a slope of -0.5 due to the expected inverse square-root dependence of the noise; the measured points are in good agreement with that expectation.

(A color version of this figure is available in the online journal.)

the $2 \mathrm{D}$ spectral images over a range slightly less than their height, to utilize pixels having similar exposure levels, to the maximum possible degree. We similarly restrict our analysis to wavelengths well above the half-intensity points on the grism sensitivity function, also to use pixels with similar exposure levels as much as possible.

2. We integrate the grism spectra over wavelength within our adopted wavelength range, and construct a band-integrated transit curve. We fit to this transit curve to obtain the whitelight transit depth $\left(R_{p}^{2} / R_{s}^{2}\right)$. We save the white-light transit depth to use below.

3. We smooth the grism spectra using a Gaussian kernel with a FWHM $=4$ pixels. This reduces the effect of undersampling. We construct a template spectrum from the out-of-transit smoothed spectra, and we shift it in wavelength, and scale it linearly in intensity, to match each individual grism spectrum, choosing the best shift and scale factors using linear least-squares. We subtract the shifted and scaled template to form residuals, and normalize the residuals by dividing by the template spectrum. This procedure removes the white-light transit, but preserves the wavelength variation in transit depth.

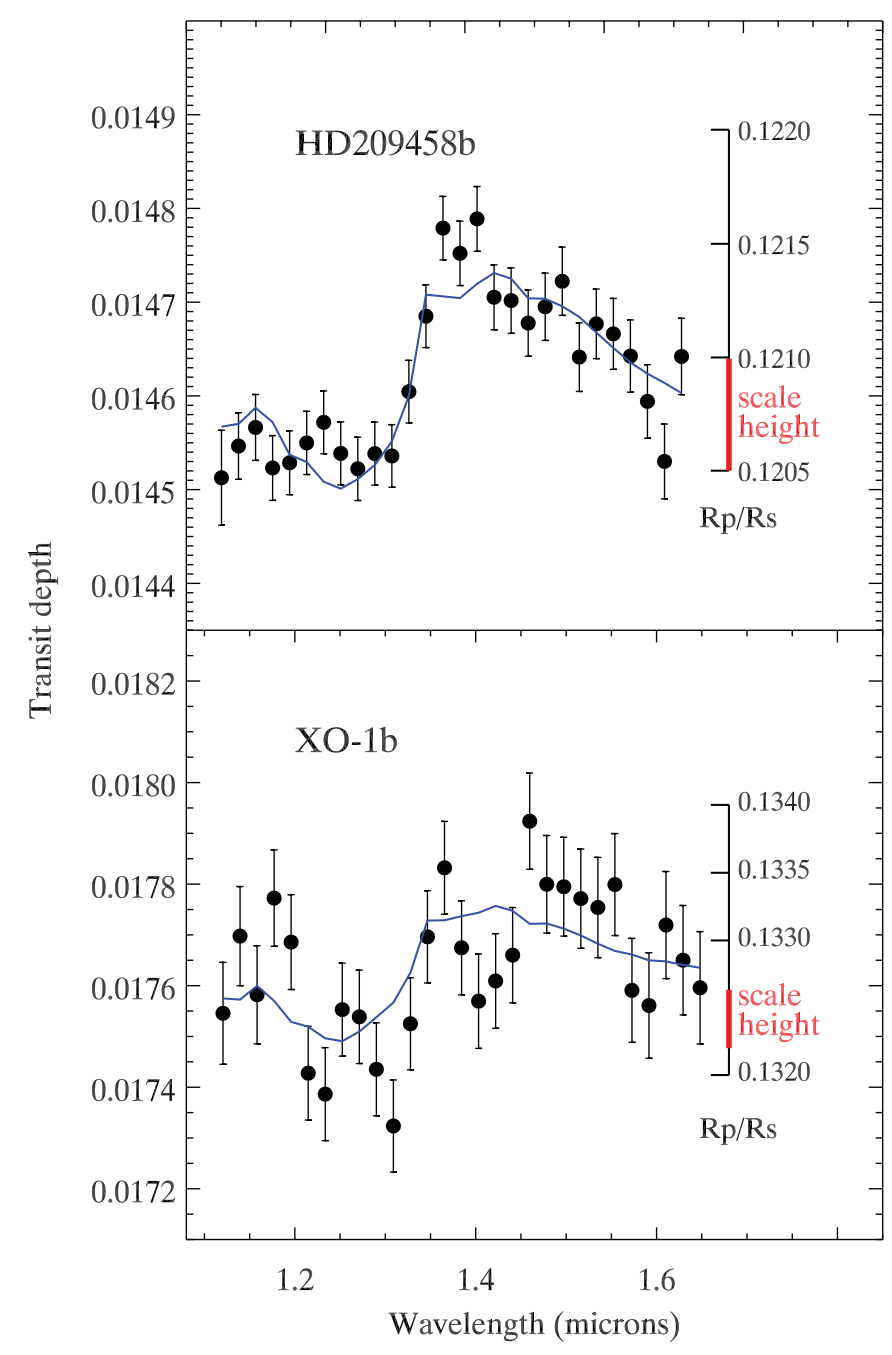

Figure 10. Our results for transmission spectra for HD 209458b and XO$1 \mathrm{~b}$ in the WFC3 bandpass, compared to models based on Spitzer secondary observations (blue lines). The spectral resolving power of these measurements is $\lambda / \delta(\lambda) \approx 70$. The amplitude of the $1.4 \mu \mathrm{m}$ water absorption is about 200 parts per million (ppm) in both cases, but the errors are smaller for HD 209458b due to the greater photon flux. The ordinate (transit depth) is $R_{p}^{2} / R_{s}^{2}$, but $R_{p} / R_{s}$ is shown by the scale on the right, and the red bars indicate the pressure scale heights for both planetary atmospheres. The water absorption we detect is about two pressure scale heights.

(A color version of this figure is available in the online journal.)

4. At each wavelength, we fit a transit curve to the residuals as a function of time, accounting for the wavelength dependence of stellar limb darkening. We add the amplitude of this transit curve (a "differential amplitude") to the depth of the white-light transit from above. We then coadd the results in groups of four wavelengths (columns on the detector) to match the smoothing described above. The result is the exoplanetary transmission spectrum.

5. We determine errors using a residual-permutation method, comparing those to errors calculated by binning the residuals over increasing time intervals (to verify an inverse square-root dependence), and by comparing to an ab initio estimate of the photon noise.

6. We verify the sensitivity of the method to assure that it does not numerically attenuate the exoplanetary spectrum. We inject numerically an artificial spectrum into the data at the earliest practical stage of the analysis, and we recover it at the correct amplitude. 


\section{RESULTS FOR TRANSMISSION SPECTRA}

\subsection{Comparison to Expectations from Spitzer}

We here illustrate our observed spectra, and immediately compare them to models that are consistent with Spitzer emergent intensity observations of these planets. The strong similarity between observed and modeled spectra reinforces our conclusion (Section 6.1) that we are observing real exoplanetary absorption. In Section 6, we explore comparisons with model atmospheres in more depth.

Figure 10 overplots modeled transmission spectra for each planet in comparison to our observed results. The modeled spectra were calculated by Adam Burrows (Burrows et al. 2001, 2010; Howe \& Burrows 2012) based on combining day- and night-side model atmospheres that are consistent with Spitzer secondary eclipse observations for these two planets (Burrows et al. 2007; Machalek et al. 2008). The day- and night-side model atmospheres were combined by equalizing their basal pressures to join them at the terminator of the planet. The transmittance spectrum used for Figure 10 represents a line of sight that passes through both the day- and night-side models. We fit the modeled absorptions to the data by scaling them in amplitude, and offsetting them slightly in overall radius, but not changing the modeled shape. The fitted amplitude of the HD 209458b absorption is 0.57 of the modeled value, and for $\mathrm{XO}-1 \mathrm{~b}$ the fitted amplitude is 0.84 of the model. These factors are physically reasonable, as we discuss below, and the correspondence between the observed and modeled shape of the absorptions is clear.

Our analysis uses simple procedures without recourse to an instrument model, and the smoothing we implement is motivated by an effect that we understand physically (the undersampling). Our errors are close to photon-limited as verified by the inverse square-root dependence when bining, and by the comparison with the prayer-bead errors. We are therefore certain that Figure 10 represents the real astrophysical absorption spectrum, especially in the case of HD 209458b, where the amplitude of the absorption $(200 \mathrm{ppm})$ is more than five times the average error per point, and the absorption is sampled by many observed points.

In the case of XO-1b, the detection is less visually obvious than for HD 209458b, but is still robust. If we fit a flat line (the no absorption case) to the XO- 1 b observations, the $\chi^{2}$ is 64.6 for 28 degrees of freedom. That rejects the flat line at greater than $99 \%$ confidence. Moreover, the total context of the observations, including the similarity to both the model and the HD 209458b observations, allows us to conclude that real astrophysical absorption is observed in XO-1b as well as in HD 209458b.

In Section 6.1 we consider whether stellar activity could contribute significantly to our derived exoplanetary spectra, or whether true wavelength-dependent absorption in the planetary atmospheres is dominant, and we conclude the latter. A more elaborated comparison with planetary models is presented in Section 6.

\subsection{Comparison to Previous Observational Results}

The absorptions we detect are considerably weaker than claimed by several previous investigations. The clearest discrepancy is for XO-1b, as illustrated on Figure 11. This repeats our XO-1b spectrum, overlaid on the same plot as the results from Tinetti et al. (2010) and Crouzet et al. (2012). The large water absorption derived by Tinetti et al. (2010) is inconsistent with our results: such a large signal would be obvious even at

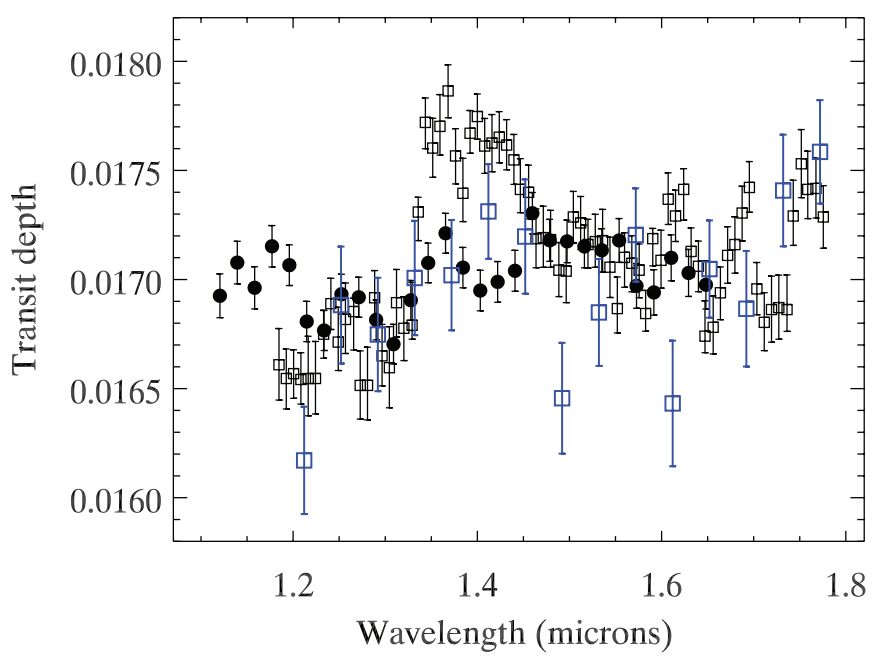

Figure 11. Comparison of our transmission spectrum of XO-1b (solid points; see Figure 10) with the NICMOS results from Tinetti et al. (2010; black squares) and Crouzet et al. (2012; blue squares). (Our data have been offset in transit depth for clarity.) Our WFC3 water absorption is of much smaller amplitude than seen in the NICMOS data (see text for discussion).

(A color version of this figure is available in the online journal.)

the Figure 5 stage of our analysis. Crouzet et al. (2012) concluded that NICMOS instrumental signatures remain comparable with the expected amplitude of molecular features, even after a decorrelation analysis. Crouzet et al. (2012) derived significantly larger errors than Tinetti et al. (2010), and we therefore find less disagreement with the Crouzet et al. (2012) spectrum. The discrepancy between Tinetti et al. (2010) and our result is either due to the intractability of NICMOS instrument signatures, or to variability in the exoplanetary atmosphere (i.e., clouds at the terminator).

If our difference with the Tinetti et al. (2010) results is due to variable clouds at the planet's terminator, it is informative to convert the required change in absorption to an equivalent number of opaque scale heights. Tinetti et al. (2010) derive an absorption depth in the spectrum of approximately $1150 \mathrm{ppm}$ (from 1.28 to $1.38 \mu \mathrm{m}$ ), whereas we measure only $\sim 200 \mathrm{ppm}$. In terms of equivalent planetary radii, the difference of $950 \mathrm{ppm}$ implies

$$
2 \delta R_{p} / R_{p}=0.00095 /\left(R_{p}^{2} / R_{s}^{2}\right)
$$

and adopting $R_{p}^{2} / R_{s}^{2}=0.017$ for XO-1b, we find

$$
\delta R_{p}=0.0279 R_{p}=2360 \mathrm{~km} \text {. }
$$

Therefore, a ring of height $2360 \mathrm{~km}$ would have to be opaque with clouds during our measurement but sufficiently clear to allow water absorption at the time of the NICMOS observation. The scale height, $k T / \mu \mathrm{mg}$ is about $260 \mathrm{~km}$, if we adopt $T=1200 \mathrm{~K}$ from Machalek et al. (2008) and use a molecular hydrogen composition $(\mu=2.32)$. In order to attribute our difference with Tinetti et al. (2010) to variability of the planetary absorption, requires variable clouds around the entire terminator of the planet extending over nine scale heights. We regard this as highly unlikely from a meteorological point of view. So we conclude that NICMOS exoplanet spectroscopy is unreliable when analyzed using a standard linear basis model decorrelation (e.g., Swain et al. 2008b). In this respect we concur with previous conclusions by Gibson et al. (2011) and Crouzet et al. (2012).

As regards HD 209458b, our water absorption appears inconsistent with the results of Barman (2007). Barman mentions 
that his baseline model, which accounts for water absorption he identified near $1 \mu \mathrm{m}$, predicts a peak in $R_{p}$ of $1.343 R_{\mathrm{J}}$ at $1.4 \mu \mathrm{m}$. Estimating the continuum level at $\sim 1.315 R_{\mathrm{J}}$ from Barman's Figure 1, we project that his baseline model would predict a $1.4 \mu \mathrm{m}$ absorption of about $580 \mathrm{ppm}$ - about three times what we measure. We revisit the comparison to Barman (2007) in Section 6.5.

Using Spitzer transit photometry, Beaulieu et al. (2008) found evidence for water absorption in HD 209458b. While our observations do not overlap the Spitzer bandpasses, the Beaulieu et al. (2008) band-to-band transit depth differences require stronger water absorption than we observe, by several times.

\section{INTERPRETATION USING MODEL ATMOSPHERES}

We now address the degree to which our results can be affected by stellar activity (star spots, Section 6.1), then we turn to the interpretation of our results using model planetary atmospheres. We begin model interpretation by implementing a new fast-calculation transmission model, and validate it (Section 6.2). We also re-analyze the HST/STIS optical transmittance data for HD 209458b from Knutson et al. (2007b; Section 6.3), in order to combine those data with our WFC3 results. We then implement our new transmittance code (Section 6.4), and then we compare both the HST/STIS and $H S T /$ WFC3 transmittance spectra to calculations from other models (Section 6.5).

\subsection{Effect of Star Spots}

Prior to discussing our results in terms of exoplanetary atmospheric transmission, it is necessary to demonstrate that our derived exoplanetary spectra are unlikely to be contaminated by stellar activity. Although no distinct crossings of star spots are apparent in Figure 3, two effects are possible in principle. First, crossings of multiple small spots might occur, and could produce a significant cumulative effect on the transit spectra. Second, the signatures of uncrossed star spots-not occulted by the planet during transit—could be "amplified" by the transit phenomenon. We first consider the latter effect, i.e., possible amplification of uncrossed star spots.

We know from solar observations that the cool umbrae of large sunspots exhibit water vapor absorption (Wallace et al. 1995). So the spectra of solar-type stars will exhibit $1.4 \mu \mathrm{m}$ water absorption, albeit at a very low level. When a planet transits without crossing spot umbrae, it increases the relative fraction of the unocculted disk that contains spot umbrae, hence it increases the relative depth of stellar water absorption during transit. It can be shown that the magnitude of the effect masquerading as exoplanetary transit spectra is closely approximated as $A \delta \epsilon$, where $A$ is the fractional coverage of the stellar disk by umbrae, $\delta$ is the relative absorption depth of the water band in the umbral spectrum, and $\epsilon$ is the transit depth.

We first estimate the magnitude of $\delta$ in the above expression. Ideally, we would measure $\delta$ from observed spectra of sunspots at $1.4 \mu \mathrm{m}$, but we do not possess such spectra due to poor telluric transmittance. From models of cool stars (Allard et al. 2000), we estimate that sunspot umbrae would exhibit about $30 \%$ relative absorption (line core to continuum) in the $1.4 \mu \mathrm{m}$ water band. We check that estimate from other properties of sunspots. From umbral spectra measured in the red-optical continuum (0.87 $\mu \mathrm{m})$, Penn \& MacDonald (2007) found that umbrae are about 0.39 as intense as the surrounding photosphere, averaged over the solar cycle (umbrae are slightly darker at solar maximum, about 0.35 of the photosphere). Converting this ratio to a brightness temperature, we obtain $T_{c}=4380 \mathrm{~K}$ for the umbral continuum. We estimate the temperature in the water line-forming region as $T_{\text {water }}=3200 \mathrm{~K}$, from the molecular rotational temperature of the water lines (Wallace et al. 1995; Tereszchuk et al. 2002). Applying those brightness temperatures to $1.4 \mu \mathrm{m}$, we estimate that the umbral IR continuum intensity is about 0.52 of the photosphere, and the water band core would be about 0.21 of the photospheric intensity if it were optically thick. That is about a factor of two stronger than we estimated from the cool star models (Allard et al. 2000), which is reasonable. We conservatively adopt the greater (i.e., optically thick) value. The amplitude of the water band in the umbral spectrum is about $\delta=0.31(0.52-0.21)$ of the photospheric intensity.

HD 209458 and XO-1 are indicated to have average activity levels in the compilation of Knutson et al. (2010). About half of solar-type stars in the Kepler sample are more active than the Sun based on variations in optical light (Basri et al. 2010). We therefore conclude that the Sun is a reasonable analogue for HD 209458b and XO-1, and we calculate the average value for coverage of the solar disk by spot umbrae. We use the monthly compilation of sunspot areas since 1874 given by Marshall Space Flight Center. ${ }^{18}$ Averaging these data, we find that the fractional solar disk coverage by sunspots (including their penumbrae) is $268 \mathrm{ppm}$ on average. At the maximum of the solar activity cycle the typical coverage value is about $3000 \mathrm{ppm}$, more than an order of magnitude larger. About $32 \%$ of the spot area is due to umbrae, based on sunspot studies (Brandt et al. 1990). Therefore the umbral disk fraction $A$ is typically $82 \mathrm{ppm}$, increasing to $960 \mathrm{ppm}$ at the strongest solar maxima. Umbral areas can be difficult to estimate, due to scattered light and limited spatial resolution. The seminal work of Howard et al. (1984) found a significantly lower (300 ppm) disk coverage at solar maximum based on Mt. Wilson photographic plates (1921-1982), but about the same average coverage that we infer here.

For HD 209458b, we have $\epsilon=0.0146$ (Knutson et al. 2007b), so $A \delta \epsilon$ is $0.4-4.3 \mathrm{ppm}$, and the numbers for XO- 1 are similarly small. Even if these stars are several times more active than the Sun at solar maximum, the amplification-of-star-spots effect is not a major contributor to our measured water band depths.

We now turn to the possible cumulative effect of crossing small star spots during transit. Occultation of water absorption in star spots would produce an apparent emission in the derived exoplanetary spectrum, or it would weaken real exoplanetary absorption. The worst case effect would occur for a star at the peak of its activity cycle, when all of the spots on the stellar disk were occulted by the planet during the specific phases of our WFC3 data (Figure 3). The star spots would have to be distributed so that they were all crossed during our partial coverage of the transit, and their sizes would have to be sufficiently equal to prevent a single large spot-crossing being visible. In this unlikely case, the planetary absorption could be weakened by $0.31(960)=298 \mathrm{ppm}$, a significant effect. However, if 960 ppm of star spot umbrae were occulted during transit, then the white-light transit in Figure 3 would be more shallow because the umbral continuum is fainter than the photosphere. That effect would be approximately $960(0.52)=$ $500 \mathrm{ppm}$ in transit depth. It would decrease our value of $R_{p} / R_{s}$ by approximately 0.002 for both HD $209458 \mathrm{~b}$ and XO-1b. Those

\footnotetext{
18 http://solarscience.msfc.nasa.gov/greenwch.shtml
} 
decreases are unlikely, given our agreement with Spitzer IR transits of HD 209458b (Crossfield et al. 2012), and with an independent transit of XO-1b analyzed by Burke et al. (2010) (Section 4.1). Moreover, if the weak absorptions we derive for these exoplanets are due to masking by star spots of intrinsically stronger exoplanetary absorption, then the specific and unlikely spot-occultation circumstances described above would have to apply independently to both stars.

We conclude that neither occulted nor unocculted star spots have significantly affected our results, and we are measuring real exoplanetary water in transmission.

\subsection{Validation of a Spectral Transmittance Code}

In Section 6.3, we interpret our results using a new spectral transmittance code. This code is intended for rapid line-byline calculation of transmittance spectra, so as to explore parameter space when varying mixing ratios, cloud heights, atmospheric temperature, etc. We intend to use it for future WFC3 investigations as well as to illuminate the present results. Here, we describe the code, and the tests we have conducted to validate it.

Our transmittance code is based on the work of Richardson et al. (2003); consequently many of the algorithms it uses were tested previously. We have modified the original code to use the slant-path geometry appropriate for transmittance spectra. The code uses a layered hydrostatic equilibrium atmosphere, with pressures spaced equally in the $\log$ from 1 to $10^{7}$ dynes $\mathrm{cm}^{-2}$ (adjustable). It incorporates continuous opacity due to collisioninduced absorption by $\mathrm{H}_{2}$ (Zheng \& Borysow 1995; Borysow 2002), and it includes a provision for calculating number densities of major molecules in thermal equilibrium. Nevertheless, we here specify a depth-independent mixing ratio of water in an ad hoc fashion for the purpose of exploring parameter space. We include opacity for water using the line-by-line data from Partridge \& Schwenke (1997). To speed the calculations, we sum and pre-tabulate the strengths of the extremely numerous water lines within wavelength bins, and represent each bin by a single line having the strength of the total. We choose a bin width of $0.1 \mathrm{~cm}^{-1}\left(2 \times 10^{-5} \mu \mathrm{m}\right.$ at $\left.1.4 \mu \mathrm{m}\right)$, much smaller than our WFC3 resolution. The average within each bin is represented by a Voigt profile with a damping coefficient of $0.1 \mathrm{~cm}^{-1}$ per atmosphere. This averaging is valid only at a single temperature, because each bin in wavelength will contain lines of different lower state excitation, which adds a nonlinear factor. We thus pre-tabulate a separate line list for each isothermal model. From the line and continuous opacity, we numerically integrate along the un-refracted path that passes tangent to each layer, to calculate the optical depth $\tau$ and extinction $\exp (-\tau)$ over that path. We adopt a source function of zero, i.e., we neglect self-emission by the planetary atmosphere. From the optical depths, we calculate the effective blocking area of the planet as a function of wavelength.

To validate this code, we conducted two tests. First, we specified an ad hoc continuous opacity proportional to $\lambda^{-4}$, and we conducted a test described by Shabram et al. (2011). This test involves verifying the analytic relation $d R_{p} / d \ln \lambda=-4 H$, where $R_{p}$ is the calculated wavelength-dependent transit radius, and $H$ is the pressure scale height. Using a 1000 layer isothermal atmosphere with a constant scale height, we calculated the slope of $R_{p}$ versus $\ln \lambda$, and we find agreement with the slope of -4 to within $0.58 \%$. For atmospheres having 500 and 200 layers, our precision on the slope is 1.28 and $3.42 \%$, respectively. Our grid of spectral calculations to interpret our WFC3 spectra

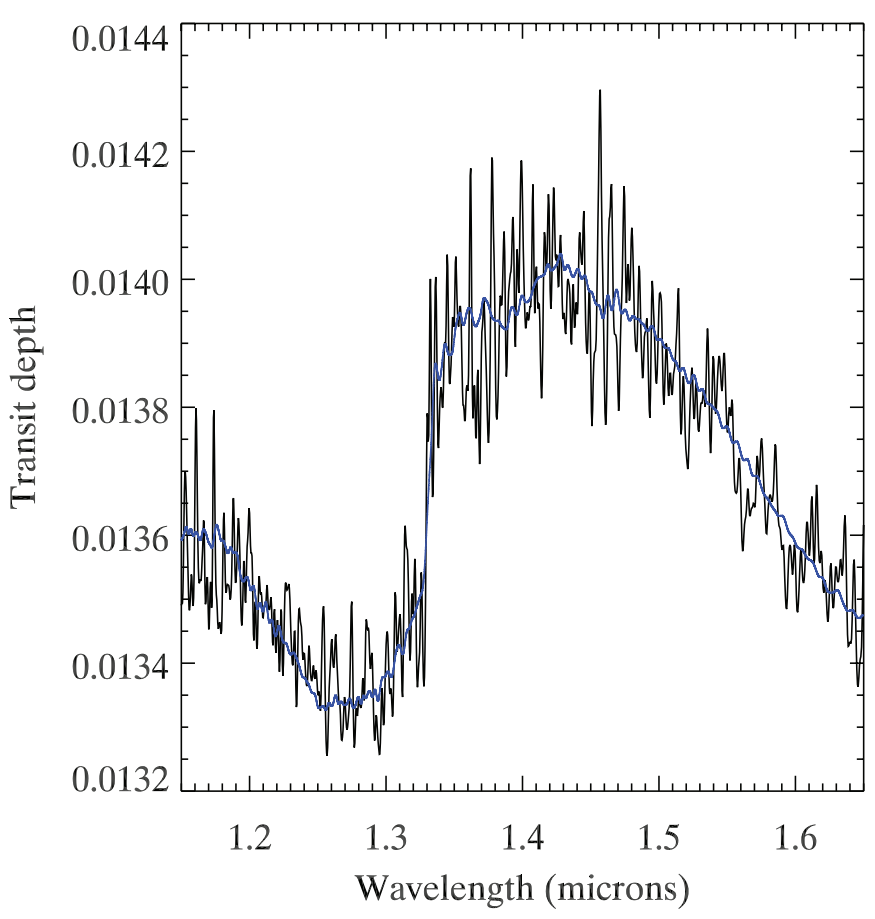

Figure 12. Validation of our transmission spectral model (blue line) vs. an independent calculation from Fortney et al. (2010) for an isothermal model at $1500 \mathrm{~K}$.

(A color version of this figure is available in the online journal.)

(Section 6.3) uses the 200 layer version, which is more than adequate for the present purpose, as our second test now establishes.

For the second test, we compared our calculated water transmission spectrum to an independent calculation from J. J. Fortney. The Fortney model is new, but uses the methods described in Fortney et al. (2010). This comparison used our 200 layer version of the code, and an isothermal model having $T=1500 \mathrm{~K}$, a surface gravity of 10 meters $\mathrm{s}^{-2}$, and a water mixing ratio (independent of pressure) of 0.00045 . The Fortney calculation uses different algorithms, different layering, and different numerical approximations to represent the same atmosphere. Results from the two calculations are in excellent agreement, and shown in Figure 12, where the (smoothed) results from the new code track the Fortney calculation very closely. As an additional test, we repeated the Figure 12 comparison using a calculation by N. Madhusudhan, adopting different atmospheric parameters, and we again achieved excellent agreement (not illustrated here). We therefore conclude that our new transmittance code is validated for the purpose of this paper.

\subsection{Re-analysis of the HST/STIS Photometry}

We want to utilize our results together with transmission spectra derived by Knutson et al. (2007b) from HST/STIS data. Knutson et al. (2007b) give errors for $R_{p}$ and $R_{s}$ separately, but a large fraction of those errors are common-mode between $R_{p}$ and $R_{s}$. To clarify the error on $R_{p} / R_{s}$ from the STIS data, we were motivated to re-analyze that photometry (from Table 1 of Knutson et al. 2007b). Our re-analysis adopts many of the Knutson et al. (2007b) parameters without attempting to vary them. Specifically, we adopt their transit center times, their nonlinear limb darkening coefficients, and their band-to-band differences in $R_{p} / R_{s}$. We fit their photometry for all 10 bands simultaneously, using a Markov Chain Monte Carlo (MCMC) 
method with Gibbs sampling (Ford 2005), to determine the band-averaged value of $R_{p} / R_{s}$, as well as $a / R_{s}$ and $i$. We initialize our MCMC chains using the parameters from Knutson et al. (2007b), and these good starting values allow our chains to converge rapidly (in approximately 1200 steps). However, the chains run slowly, because we are fitting to 10 bands simultaneously using nonlinear limb darkening. We therefore run four independent chains simultaneously, each having 60,000 steps, and we combine their posterior distributions. The combined distributions are closely approximated by Gaussians, and yield $R_{p} / R_{s}=0.1210 \pm 0.0001$. The small error in $R_{p} / R_{s}$ reflects the large volume of data over 10 wavelength bands.

Our re-determined value for the band-averaged $R_{p} / R_{s}$ is in mild disagreement with Knutson et al. (2007b), in the sense that our result favors a larger $R_{p}$ by 1.5 times their error for $R_{p}$. However, our re-determined STIS value for $R_{p} / R_{s}$ is in good agreement with our WFC3 value, so our analysis is selfconsistent. As noted in Section 4.1, we also agree with other IR determinations of $R_{p} / R_{s}$ (Burke et al. 2010; Crossfield et al. 2012), except for the values from Beaulieu et al. (2008).

\subsection{Interpretation of HD $209458 b$ Using Model Atmospheres}

We now turn to exploring what our new observations imply about the atmospheres of these planets. In the case of XO-1b, the scaling factor required to fit the Spitzer-based model to the data on Figure 10 was 0.84, versus 0.57 for HD 209458b. These factors do not per se indicate discrepancies with the conclusions from the Spitzer investigations (Knutson et al. 2008; Machalek et al. 2008), because molecular absorption during transit is much more sensitive to conditions such as clouds and haze. Given that the scale factor for $\mathrm{XO}-1 \mathrm{~b}$ is close to unity, and considering the larger noise level of that spectrum compared to HD 209458b, we conclude that $\mathrm{XO}-1 \mathrm{~b}$ is adequately described by extant models, not counting the Tinetti et al. (2010) calculations. In contrast, the relatively small scale factor required for HD 209458b, combined with the lower noise level of those data, motivate us to inquire further what our results imply for the atmosphere of that planet.

In principle, it would be possible to analyze our water transmission spectrum simultaneously with sodium absorption measurements (Charbonneau et al. 2002; Sing et al. 2008; Snellen et al. 2009), CO absorption data (Snellen et al. 2010), and Spitzer secondary eclipse photometry (Knutson et al. 2008) and spectroscopy (Richardson et al. 2007; Swain et al. 2008a). Such an analysis could incorporate guidance from hydrodynamic models (Showman et al. 2008; Dobbs-Dixon et al. 2012; Showman et al. 2013; Rauscher \& Menou 2013) to account for the longitudinal transfer of stellar irradiance, and could explore the full parameter space of composition and temperature structure (Madhusudhan \& Seager 2009, 2010), as well as the effect of clouds and hazes at the terminator (Parmentier et al. 2013). However, we do not attempt such an ambitious investigation here. Instead, we compare the combination of HST/STIS and HST/ WFC3 measurements to theoretical transit spectra from various models without much fine-tuning, and we discuss the discrepancies so as to guide the general direction of more exhaustive atmospheric modeling in the future.

The weakness of the water band we observe is reminiscent of the first detection of this (indeed, of any) exoplanetary atmosphere by Charbonneau et al. (2002) who observed the sodium "D" line doublet near $0.5893 \mu \mathrm{m}$ using HST/STIS. To account for the weakness of the sodium feature, Charbonneau et al. (2002) varied the height of an opaque cloud layer, using the model described by Brown et al. (2001). We here perform

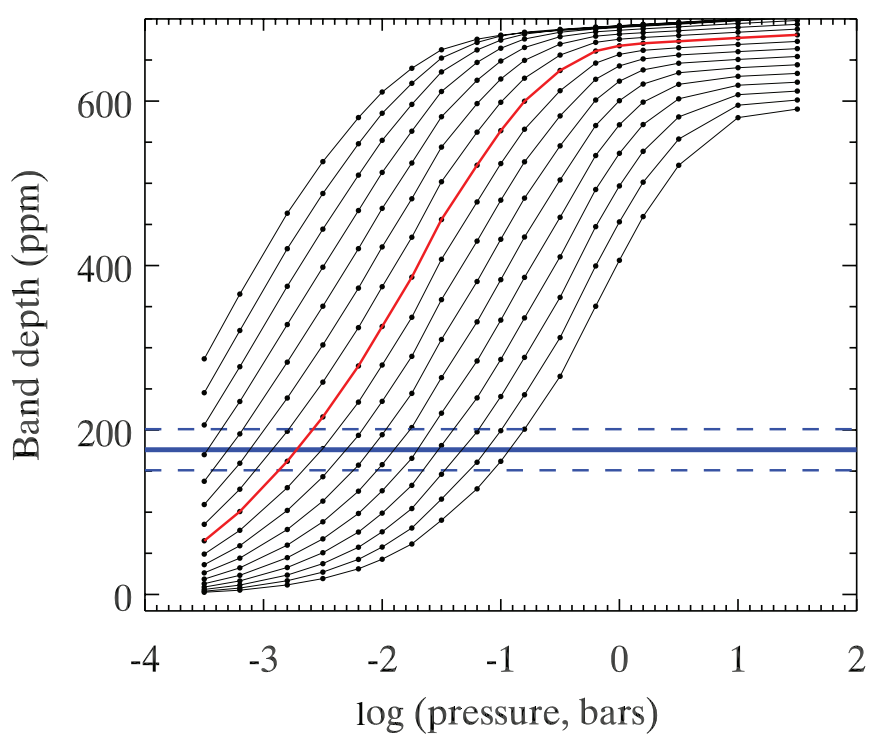

Figure 13. Depth of the $1.4 \mu \mathrm{m}$ water band vs. cloud top pressure for an isothermal model at $1200 \mathrm{~K}$. Lines represent different mixing ratios of water. From top to bottom the log of the mixing ratios vary from -2.0 to -5.2 in increments of -0.2 . The red contour is the mixing ratio expected for solar abundance. Small black points are the actual calculations from our transmittance model. The blue line is our observed band depth of $176 \mathrm{ppm}$, with $\pm 1 \sigma$ errors (dashed lines). For this figure, band depth is defined as the average transit depth from 1.36-1.44 $\mu \mathrm{m}$ minus the average depth from 1.27-1.30 $\mu \mathrm{m}$.

(A color version of this figure is available in the online journal.)

a similar calculation, using our spectral transmittance code described in Section 6.2. We vary both the pressure level for the top of an opaque cloud layer, and the mixing ratio of water vapor, and we measure the strength of the $1.4 \mu \mathrm{m}$ band at about 300 points over a 2D grid, calculating a full transmittance spectrum (as per Figure 12) at each grid point. The question of the temperature structure at the terminator of the planet-needed for this calculation - is problematic, in spite of significant work on this topic (Sing et al. 2008). Our results are not very sensitive to the $T(P)$ relation, so we prefer to use a simple isothermal atmosphere, based on the observed brightness temperatures from Spitzer (Crossfield et al. 2012). Ideally, we would average the day-side and night-side brightness temperatures from Spitzer to arrive at an estimate of the temperature at the terminator. Although Spitzer around-the-orbit observations for HD 209458b have been obtained, they are still under analysis. Therefore we adopt the same relative day-to-night change as for HD 189733b (Knutson et al. 2007a), and apply that relative variation to the HD 209458b day side temperature from Crossfield et al. (2012). This yields an estimate of $1200 \mathrm{~K}$ for the terminator, and Figure 13 shows a grid of band amplitudes calculated at that temperature.

The blue line with $\pm 1 \sigma$ error limits on Figure 13 is the amplitude of the observed band. Since the models have finescale structure with wavelength, and the observations have point-to-point noise, we define a band amplitude for Figure 13 by averaging over two wavelength regions having low and high water vapor opacity, respectively, i.e., 1.27-1.30 and 1.36-1.44 $\mu \mathrm{m}$. This results in an observed band amplitude of $176 \pm 25 \mathrm{ppm}$. Note that the amplitude of the absorption near the actual bandhead at $1.38 \mu \mathrm{m}$ is slightly higher, about $200 \mathrm{ppm}$.

Contours of constant mixing ratio are included on Figure 13. The solar-abundance contour (drawn in red) intersects the observed band amplitude only where the cloud top pressure is low, near 1.5 mbar. That is similar to, but less extreme than, 


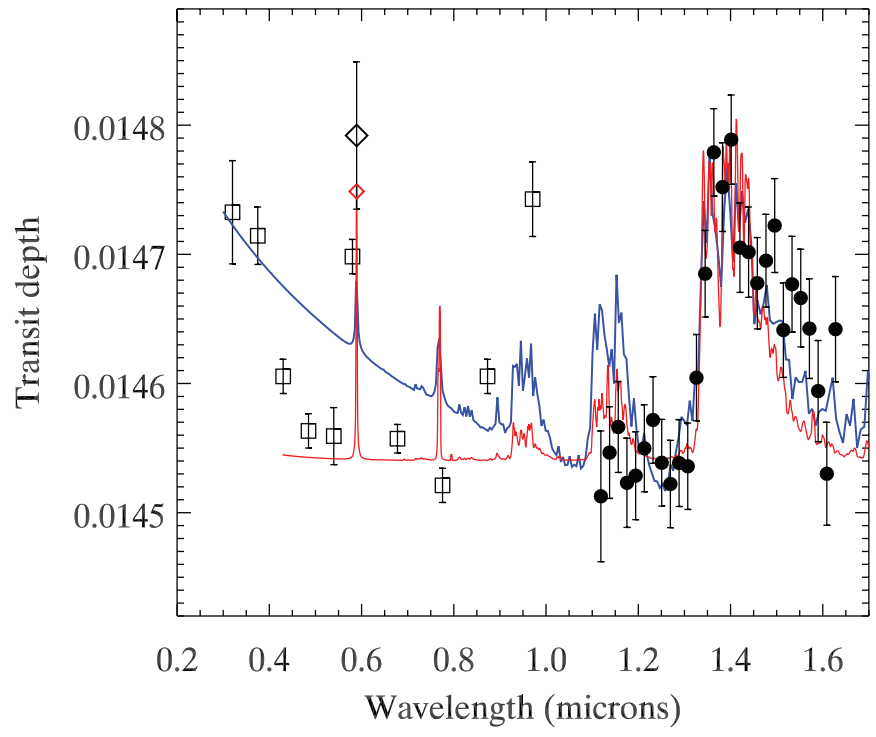

Figure 14. Transmission spectrum of HD 209458b derived from HST spectroscopy. Our WFC3 results are the solid points. The open squares are our re-analysis of the STIS bands defined by Knutson et al. (2007b), and the diamond is the narrow sodium band absorption from Charbonneau et al. (2002). The red line is the transmittance spectrum from an isothermal Burrows model, having an extra opacity of gray character and magnitude $0.012 \mathrm{~cm}^{2} \mathrm{~g}^{-1}$. The red diamond integrates the red model over the sodium bandpass. The blue line is a Dobbs-Dixon model for HD 209458b, with no gray opacity, but with $\lambda^{-4}$ (Rayleigh) opacity, normalized to magnitude $0.001 \mathrm{~cm}^{2} \mathrm{~g}^{-1}$ at $0.8 \mu \mathrm{m}$. Because the blue model has no gray opacity, we scale-down the modulation in this spectrum by a factor of three for this comparison (see text).

(A color version of this figure is available in the online journal.)

the sodium case; Charbonneau et al. (2002, p. 383) remarked that if the sodium weakness is attributed solely to clouds, then it "would require ... cloud tops above 0.4 mbar." Fortney et al. (2003) concluded that silicate and iron clouds could reside at pressures of several millibars in the atmosphere of HD 209458b. As concerns alternate explanations, note that Figure 13 implies a semi-forbidden region, where no contours pass into the lower right of the plot. The cloud-top pressure levels depicted on the right of Figure 13 imply a clear atmosphere, and even our lowest modeled mixing ratio $(-5.2$ in the $\log )$ is not sufficient to weaken the band to account for our observations if the atmosphere is clear. The total column density along the line of sight at high pressures in the tangent geometry is so large that even unrealistically small mixing ratios are insufficient to weaken the band to the observed degree, in the absence of other water-destruction mechanisms such as photolysis (unlikely in the deep atmosphere). We conclude that we are not observing a clear atmosphere.

Figure 14 shows our full HD 209458b transit depth spectrum $\left(R_{p}^{2} / R_{s}^{2}\right)$, combining both our WFC3 and re-analyzed STIS results. The combination of these data span wavelengths from 0.2 to $1.6 \mu \mathrm{m}$ with a consistent observed lower envelope and overall level in $R_{p}^{2} / R_{s}^{2}$. We first add the caveat that systematic differences might still remain between the overall level of the STIS and WFC3 transit depths, in spite of the seeming consistency. Nevertheless, Figure 14 represents the best composite optical/near-IR transmission spectrum of HD 209458b to date, so we proceed to ask what it reveals about the exoplanetary atmosphere.

Now, we compare this combined spectrum to two models. First, we used a grid of spectra by Adam Burrows, based on a
$1200 \mathrm{~K}$ isothermal temperature structure, as used above. The grid utilizes the methodologies described by Burrows et al. (2001) and Burrows et al. (2010) and Howe \& Burrows (2012), but it incorporates different amounts of extra gray opacity. We interpolate in this grid to find that an extra opacity of $0.012 \mathrm{~cm}^{2} \mathrm{~g}^{-1}$ matches the $1.4 \mu \mathrm{m}$ water absorption at the bandhead, and provides suitably low absorption at $1.15 \mu \mathrm{m}$. The lowering of the $1.15 \mu \mathrm{m}$ absorption occurs because that intrinsically weaker band requires a longer path length to produce significant absorption, and long path lengths are masked by the extra opacity. A Burrows isothermal model having no extra opacity (not illustrated) shows a much more prominent peak at $1.15 \mu \mathrm{m}$.

The profile of the $1.4 \mu \mathrm{m}$ band is not matched optimally by the isothermal models, not as well as on Figure 10 for example. The real absorption line of sight passes through different temperatures on day and night hemispheres of the planet. Figure 10 accounts (crudely) for different temperatures along the line of sight, not included in the isothermal model for Figure 14. Including that line-of-sight temperature variation may be essential to matching the band profile.

The Burrows model on Figure 14 is sufficiently highresolution in wavelength to permit meaningful comparison with the sodium absorption measured by Charbonneau et al. (2002) and Snellen et al. (2009). We plot the "narrow" band absorption from Charbonneau et al. (2002) on Figure 14 (triangle point with error bar). The Snellen et al. (2009) results (not plotted) are consistent with Charbonneau et al. (2002), considering the different bandpasses. Integrating the Burrows model having $0.012 \mathrm{~cm}^{2} \mathrm{~g}^{-1}$ extra opacity over the band used by Charbonneau et al. (2002; red diamond point) produces agreement within the error bar.

One aspect of the observations that are not reproduced by the simple isothermal Burrows model with gray opacity is the tendency toward increasing radius in the blue and UV, at the left edge of Figure 14. An increase of transit radius at short wavelength may be related to the absorber that causes a temperature inversion in this planet (Burrows et al. 2007). It may also be produced by Rayleigh scattering from a population of small particles, which we do not include in the Burrows calculations for Figure 14 (but Rayleigh scattering by molecules is included). In this regard, we overplot a model from Ian DobbsDixon (blue line on Figure 14), based on the methods described in Dobbs-Dixon et al. (2012). This model uses a full radiative hydrodynamic treatment of the temperature structure, which may explain why it produces a better (but not perfect) account of the $1.4 \mu \mathrm{m}$ band profile. It has no extra gray opacity, but it incorporates extra opacity of $0.004 \mathrm{~cm}^{2} \mathrm{~g}^{-1}$ at $0.8 \mu \mathrm{m}$, with a $\lambda^{-4}$ dependence. Because that Rayleigh opacity is concentrated at short wavelengths, we must scale the modulation in the modeled spectrum downward by a factor of three to match the observed $1.4 \mu \mathrm{m}$ band. That scaling is unphysical, but it allows us to judge the relative importance of gray versus Rayleigh opacity that will be needed to match the observations. After scaling, the blue line produces relatively good agreement with the $1.4 \mu \mathrm{m}$ data, but overestimates the $1.15 \mu \mathrm{m}$ feature as well as slightly overestimating the increase in absorption in the blue and UV.

Finally, we point out one notable discrepancy in the model comparisons. The STIS point near $0.95 \mu \mathrm{m}$ cannot be reproduced by models while still being consistent with our $1.4 \mu \mathrm{m}$ band measurement. Barman (2007) argued for water vapor in HD 209458b based in part on the STIS data near $0.95 \mu \mathrm{m}$, but 
Knutson et al. (2007b) did not claim water detection from those same data.

From the above comparisons, we conclude:

1. A uniformly distributed extra opacity, gray in wavelength dependence, of approximate magnitude $0.012 \mathrm{~cm}^{2} \mathrm{~g}^{-1}$ is needed in isothermal models at $1200 \mathrm{~K}$ in order to match our observed water transit absorption for HD 209458b at $1.4 \mu \mathrm{m}$, and to produce the weakness of water at $1.15 \mu \mathrm{m}$, and account for weak sodium absorption in the optical.

2. Models that include realistic temperature distributions along the line of sight may be required to match the band profiles of the water absorption.

3. Our results are consistent with the situation described by Pont et al. (2013) for HD 189733b, wherein weak molecular absorptions are superposed on a spectrum whose broad variations with wavelength in transit are dominated by haze and/or dust opacity. However, the extra opacity required for HD 209458b needs to be grayer than the strong Rayleigh component needed for HD 189733 b.

\section{SUMMARY AND FURTHER IMPLICATIONS}

We have demonstrated that WFC3 spectroscopy using the new spatial scan mode can yield exoplanetary spectra whose error level falls significantly below the $0.01 \%$ level. We detect $1.4 \mu \mathrm{m}$ water in both HD $209458 \mathrm{~b}$ and XO-1b at a relatively low level of absorption, only $200 \mathrm{ppm}$ at the $1.38 \mu \mathrm{m}$ bandhead. Our results for XO-1b contradict the much larger absorption derived by Tinetti et al. (2010), and we concur with Gibson et al. (2011) that NICMOS spectroscopy is unreliable when analyzed using the standard linear basis model approach (Swain et al. 2008b). Fortney (2005, Abstract) predicted that giant exoplanetary atmospheres would contain condensates and hazes that would "lead to weaker than expected or undetected absorption features". For HD 209458b, an atmosphere with $0.012 \mathrm{~cm}^{2} \mathrm{~g}^{-1}$ of extra opacity, gray in character, is required in order to match the subtle water absorption we detect and the sodium absorption in the optical. Pont et al. (2013) have argued that molecular absorptions in HD 189733 b are weakened by haze and/ or dust opacity, and our results suggest a similar situation for HD 209458b. One difference is that haze and/or dust opacity is grayer for HD 209458b, with a weaker Rayleigh component as compared to HD 189733 b.

The capability to derive transmittance spectra for planets transiting bright stars, at an error level below $0.01 \%$ in a single $H S T$ visit, opens new scientific horizons. For example, it becomes feasible to monitor meteorological variability of conditions at the terminator of the planet. The transmittance spectra of super-Earths should be detectable using multiple visits (Bean et al. 2010, 2011; Kreidberg et al. 2013), even for high molecular weight atmospheres. Applying the spatial scan to secondary eclipses, it should be possible to confirm the existence of atmospheric temperature inversions via low-noise detection of the water band profile in emission rather than in absorption. Finally, we note that there is much discussion in the community concerning dedicated space missions to characterize exoplanetary atmospheres using transits. The design of such missions should prudently consider that molecular absorptions may be considerably weaker than are modeled using clear atmospheres.

We are grateful for the excellent support of the staff at STScI for the scheduling and execution of our demanding observations, especially our contact person Shelly Meyett. We also thank an anonymous referee for insightful comments that improved this paper.

\section{APPENDIX}

\section{PRODUCTION OF 2D SPECTRAL FRAMES}

We here describe how the spectral frames shown in Figure 1 are constructed from the sample-up-the ramp data frames, and how discrepant pixels and energetic particle hits are corrected. Each non-destructive sample of the detector is provided to observers as an extension in a FITS file (the *ima.fits files available from the Multi-Mission Archive at Space Telescope, MAST). Normally the *ima.fits files are processed by an analysis pipeline at the Space Telescope Science Institute (STScI), which fits the slope and makes the resulting intensities available in *flt.fits files. However, in the case where the source is rapidly moving, as it is for the spatial scan mode, the normal analysis pipeline is inapplicable because a given pixel is not always viewing the same celestial scene, and that invalidates the pipeline's fit to the slope at each pixel. In scan mode, there are two alternative methods that can be used for initial data processing. One method is to subtract the first read of the detector from the last read, thus producing an image of the total accumulated electrons on the detector during the spatial scan. However, we use an alternate method that offers several advantages over a simple "last minus first" subtraction.

For a given exposure, let $M$ be the number of times that each pixel is sampled, and let $i=1, M$ index the individual samples. For each $i \leqslant M-1$, we subtract sample $i$ from sample $i+1$, thus forming differences $D_{i}=C_{i+1}-C_{i}$ where $C$ denotes the charge on a given pixel in electrons. Because scanning occurs parallel to columns, these differences reveal the spectrum of the star over a limited range of rows on the detector. Considering the difference $D_{i}$, we zero the rows not containing the target star, using a top-hat mask whose width extends to the wings of the stellar point spread function (PSF) - typically 15 pixels ( 1 pixel $=0.14$ arcsec). We then form the sum

$$
S_{j}=\sum_{i=1}^{M-1} D_{i},
$$

where $j=1, N$ indexes the $N$ times that the scan is repeated over the duration of the transit. Each sum is the image of the spatial scan that we use in further analysis.

The above methodology reduces the effect of sky background. Because of the masking applied to each of the $D_{i}$, sky background not immediately adjacent to or underlying the star is zero-ed, and does not accumulate in the summation in Equation (1). Only the background near the star in each $D_{i}$ survives this procedure. However, the residual background flux that accumulates in the brief interval between consecutive reads of the detector is small $(<0.1 \%)$ compared to the fluxes from these bright stars, and is not removed at this stage of the analysis. It is removed by our wavelength shift correction procedure, described in Section 4.2. Removal of the residual background by that procedure produces a small bias on the stretch factor (see Section 4.2), but we verified that the bias has negligible effect (by several orders of magnitude) on the final exoplanetary transmission spectrum. The residual background is not removed when fitting to the white-light transit (Section 4.1). In that case it biases the white-light transit depths, but the magnitude of the 
bias is much less than the observational error $(<0.2 \sigma)$. Advantages of the above procedure are that it minimizes the effect of hot pixels and energetic particle events that would otherwise overlap the scan. Moreover, it allows for discrimination against other stars that are spatially resolved, but would overlap in a simple last-minus first difference.

Although the procedure described above minimizes the effects of discrepant pixels, it does not completely eliminate them. Normally, hot and transient pixels are identified and corrected via a numerical median filter applied to the time history of each pixel. The spatial scan rate variations complicate that procedure because they contribute to the intensity fluctuations of every pixel versus time, and could interfere with the median filter process. We therefore apply a five-point median filter to the ratio of a given pixel intensity to the total intensity in that row of the detector. This ratio cancels the scan rate variations (Figure 1), and isolates the behavior of the pixel itself. We apply the median filter in a two-pass process. The first pass corrects pixels that are discrepant by more than $10 \sigma$ from the median value, where $\sigma$ is the standard deviation of the difference between the time history of the pixel and the median-filtered version of that time history. The first $10 \sigma$ pass serves to eliminate very large fluctuations that might perturb the calculation of $\sigma$. The second pass uses a lower threshold, correcting pixels that are discrepant by more than $3 \sigma$. For HD $209458 \mathrm{~b}, 0.15 \%$ of the pixels are corrected by this procedure, and $0.04 \%$ in the case of XO- 1 .

\section{REFERENCES}

Allard, F., Hauschildt, P. H., \& Schwenke, D. 2000, ApJ, 540, 1005 Barman, T. 2007, ApJL, 661, L191

Basri, G., Walkowicz, L. M., Batalha, N., et al. 2010, ApJL, 713, L155

Bean, J. L., Désert, J.-M., Kabath, P., et al. 2011, ApJ, 743, 92

Bean, J. L., Miller-Ricci Kempton, E., \& Homeier, D. 2010, Natur, 468, 669

Beaulieu, J. P., Kipping, D. M., Batista, V., et al. 2010, MNRAS, 409, 963

Berta, Z. K., Charbonneau, D., Désert, J.-M., et al. 2012, ApJ, 747, 35

Borysow, A. 2002, A\&A, 390, 779

Brandt, P. N., Schmidt, W., \& Steinegger, M. 1990, SoPh, 129, 191

Brown, T. M. 2001, ApJ, 553, 1006

Burke, C. J., McCullough, P. R., Bergeron, L. E., et al. 2010, ApJ, 719, 1796

Burrows, A., Hubbard, W. B., Lunine, J. I., \& Liebert, J. 2001, RvMP, 73, 719

Burrows, A., Hubeny, I., Budaj, J., Knutson, H. A., \& Charbonneau, D. 2007, ApJL, 668, L171

Burrows, A., Rauscher, E., Spiegel, D. S., \& Menou, K. 2010, ApJ, 719, 341

Charbonneau, D., Brown, T. M., Lathan, D. W., \& Mayor, M. 2000, ApJL, $529, \mathrm{~L} 45$

Charbonneau, D., Brown, T. M., Noyes, R. W., \& Gilliland, R. L. 2002, ApJ, 568,377

Claret, A. 2000, A\&A, 363, 1081

Claret, A., \& Bloemen, S. 2011, A\&A, 529, A75

Crossfield, I., Knutson, H., Fortney, J., et al. 2012, ApJ, 752, 81

Crouzet, N., McCullough, P. R., Burke, C., \& Long, D. 2012, ApJ, 761, 7

Deming, D., Seager, S., Winn, J., et al. 2009, PASP, 121, 952

Desert, J.-M., Vidal-Madjar, A., Lecavelier des Estangs, A., et al. 2008, A\&A, 492,585

Dobbs-Dixon, I., Agol, E., \& Burrows, A. 2012, ApJ, 751, 87

Ford, E. B. 2005, AJ, 129, 1706

Fortney, J. J. 2005, MNRAS, 364, 649

Fortney, J. J., Shabram, M., Showman, A. P., et al. 2010, ApJ, 709, 1396
Fortney, J. J., Sudarsky, D., Hubeny, I., et al. 2003, ApJ, 589, 615

Gibson, N. P., Aigrain, S., Pont, F., et al. 2012a, MNRAS, 422, 753

Gibson, N. P., Aigrain, S., Roberts, S., et al. 2012b, MNRAS, 419, 2683

Gibson, N. P., Pont, F., \& Aigrain, S. 2011, MNRAS, 411, 2199

Gillon, M., Demory, B.-O., Barman, T., et al. 2007, A\&A, 471, L51

Hayek, W., Sing, D., Pont, F., \& Asplund, M. 2012, A\&A, 539, A102

Henry, G. W., Marcy, G. W., Butler, R. P., \& Vogt, S. S. 2000, ApJL, 529, L41

Howard, R., Gilman, P. A., \& Gilman, P. I. 1984, ApJ, 283, 373

Howe, A. R., \& Burrows, A. P. 2012, ApJ, 756, 176

Knutson, H. A., Charbonneau, D., Allen, L. E., Burrows, A., \& Megeath, S. T. 2008, ApJ, 673, 526

Knutson, H. A., Charbonneau, D., Allen, L. E., et al. 2007a, Natur, 447, 183

Knutson, H. A., Charbonneau, D., Noyes, R. W., Brown, T. M., \& Gilliland, R. L. 2007b, ApJ, 655, 564

Knutson, H. A., Howard, A. W., \& Isaacson, H. 2010, ApJ, 673, 526

Kreidberg, L., Bean, J., Desert, J., et al. 2013, AAS Meeting 221, \#224.03

Kuntschner, H., Bushouse, M., Kummel, M., \& Walsh, J. R. 2009, ST-ECF Instrument Science Report WFC3-2009-17 (Baltimore, MD: STScI)

Machalek, P., McCullough, P. R., Burke, C. J., et al. 2008, ApJ, 684, 1427

Madhusudhan, N., \& Seager, S. 2009, ApJ, 707, 24

Madhusudhan, N., \& Seager, S. 2010, ApJ, 725, 261

Mandel, K., \& Agol, E. 2002, ApJL, 580, L171

Mandell, A., Haynes, K., Sinukoff, E., et al. 2013, ApJ, submitted

McCullough, P. M. 2008, Instrument Science Report WFC3 2008-19 (Baltimore: Space Telescope Science Institute)

McCullough, P. M., \& MacKenty, J. 2012, Instrument Science Report WFC3 2012-08 (Baltimore: Space Telescope Science Institute)

Parmentier, V., Showman, A. P., \& Lian, Y. 2013, A\&A, submitted (arXiv:1301.4522)

Partridge, H., \& Schwenke, D. W. 1997, JChPh, 106, 4618

Penn, M. J., \& MacDonald, R. K. D. 2007, ApJL, 662, L123

Pont, F., Sing, D. K., Gibson, N. P., et al. 2013, MNRAS, 432, 2917

Ranjan, S., Charbonneau, D., Désert, J.-M., et al. 2013, ApJ, submitted

Rauscher, E., \& Menou, K. 2013, ApJ, 764, 103

Redfield, S., Endl, M., Cochran, W. D., \& Koesterke, L. 2008, ApJL, 673, L87

Richardson, L. J., Deming, D., Horning, K., Seager, S., \& Harrington, J. 2007, Natur, 445, 892

Richardson, L. J., Deming, D., Wiedemann, G., et al. 2003, ApJ, 584, 1053

Seager, S., \& Sasselov, D. D. 2000, ApJ, 537, 916

Shabram, M., Fortney, J. J., Greene, T. P., \& Freedman, R. S. 2011, ApJ, 727,65

Shannon, C. E. 1949, Proc. Inst. Radio Eng., 37, 10

Showman, A. P., Cooper, C. S., Fortney, J. J., \& Marley, M. S. 2008, ApJ, 682,559

Showman, A. P., Fortney, J. J., Lewis, N. K., \& Shabram, M. 2013, ApJ, 762 , id. 24

Sing, D. K., Désert, J.-M., Fortney, J. J., et al. 2011, A\&A, 527, A73

Sing, D. K., Huitson, C. M., Lopez-Morales, M., et al. 2012, MNRAS, 426, 1663

Sing, D. K., Vidal-Madjar, A., Lecavelier des Estangs, A., et al. 2008, ApJ, 686, 667

Snellen, I. A. G., Albrecht, S., de Mooij, E. J. W., \& Le Poole, R. S. 2008, A\&A, 487,357

Snellen, I. A. G., de Kok, R. J., de Mooij, E. J. W., \& Albrecht, S. 2010, Natur, 465,1049

Swain, M. R., Bouwman, J., Akeson, R. L., Lawler, S., \& Beichman, C. A. 2008a, ApJ, 674, 482

Swain, M. R., Vasisht, G., \& Tinetti, G. 2008b, Natur, 452, 329

Tereszchuk, K., Bernath, P. F., Zobov, N. F., et al. 2002, ApJ, 577, 496

Tinetti, G., Deroo, P., Swain, M. R., et al. 2010, ApJL, 712, L139

Waldmann, I. 2012, ApJ, 747, 12

Waldmann, I., Tinetti, G., Deroo, P., et al. 2013, ApJ, 766, 7

Wallace, L., Bernath, P., Livingston, W., et al. 1995, Sci, 268, 1155

Zheng, C., \& Borysow, A. 1995, ApJ, 411, 960 